\title{
A LOCALLY DIVERGENCE-FREE INTERIOR PENALTY METHOD FOR TWO-DIMENSIONAL CURL-CURL PROBLEMS
}

\author{
SUSANNE C. BRENNER* ${ }^{*}$ FENGYAN LI ${ }^{\dagger}$, AND LI-YENG SUNG ${ }^{\ddagger}$
}

\begin{abstract}
An interior penalty method for certain two-dimensional curl-curl problems is investigated in this paper. This method computes the divergence-free part of the solution using locally divergence-free discontinuous $P_{1}$ vector fields on graded meshes. It has optimal order convergence (up to an arbitrarily small $\epsilon$ ) for the source problem and the eigenproblem. Results of numerical experiments that corroborate the theoretical results are also presented.
\end{abstract}

Key words. curl-curl problem, Maxwell eigenproblem, locally divergence-free, interior penalty methods, graded meshes

AMS subject classification. 65N30, 65N15, 35Q60

1. Introduction. Let $\Omega \subset \mathbb{R}^{2}$ be a bounded polygonal domain. Consider the following weak curl-curl problem:

Find $\boldsymbol{u} \in H_{0}(\operatorname{curl} ; \Omega)$ such that

$$
(\nabla \times \boldsymbol{u}, \nabla \times \boldsymbol{v})+\alpha(\boldsymbol{u}, \boldsymbol{v})=(\boldsymbol{f}, \boldsymbol{v}) \quad \forall \boldsymbol{v} \in H_{0}(\operatorname{curl} ; \Omega),
$$

where $\alpha$ is a constant, $(\cdot, \cdot)$ denotes the inner product of $L_{2}(\Omega)$ (or $\left.\left[L_{2}(\Omega)\right]^{2}\right)$,

$$
H(\operatorname{curl} ; \Omega)=\left\{\boldsymbol{v}=\left[\begin{array}{l}
v_{1} \\
v_{2}
\end{array}\right] \in\left[L_{2}(\Omega)\right]^{2}: \nabla \times \boldsymbol{v}=\frac{\partial v_{2}}{\partial x_{1}}-\frac{\partial v_{1}}{\partial x_{2}} \in L_{2}(\Omega)\right\}
$$

and

$$
H_{0}(\operatorname{curl} ; \Omega)=\left\{\boldsymbol{v}=\left[\begin{array}{l}
v_{1} \\
v_{2}
\end{array}\right] \in H(\operatorname{curl} ; \Omega): \boldsymbol{n} \times \boldsymbol{v}=n_{1} v_{2}-n_{2} v_{1}=0 \text { on } \partial \Omega\right\},
$$

with $\boldsymbol{n}=\left[\begin{array}{l}n_{1} \\ n_{2}\end{array}\right]$ being the unit outer normal on $\partial \Omega$.

The curl-curl problem (1.1) is related to electromagnetic problems. For $\alpha \leq 0$, it is the weak form of the time-harmonic (frequency-domain) Maxwell equations. For $\alpha>0$, it is related to the spatial problems appearing in implicit semi-discretizations of the time-dependent (time-domain) Maxwell equations.

It is well-known $[24,25,5]$ that $H^{1}$ conforming nodal finite element methods can lead to a wrong solution of (1.1) if $\Omega$ is not convex. Many alternative approaches have been developed which include $H(\operatorname{curl} ; \Omega)$ conforming edge element methods [41, $42,13,33,38,40], H^{1}$ conforming nodal finite methods with weighted regularization $[27,29]$, the singular complement/field method $[32,5]$, and interior penalty methods $[43,36,37,35,34]$.

\footnotetext{
*Department of Mathematics and Center for Computation and Technology, Louisiana State University, Baton Rouge, LA 70803 (brenner@math.lsu.edu). The work of this author was supported in part by the National Science Foundation under Grant No. DMS-03-11790.

${ }^{\dagger}$ Department of Mathematical Sciences, Rensselaer Polytechnic Institute, Troy, NY 12180 (lif@rpi.edu). The work of this author was supported in part by the National Science Foundation under Grant No. DMS-06-52481.

$\ddagger$ Department of Mathematics, Louisiana State University, Baton Rouge, LA 70803 (sung@math.lsu.edu).
} 
In this paper we take a different approach. By the Helmholtz decomposition, the solution $\boldsymbol{u} \in H_{0}(\operatorname{curl} ; \Omega)$ of (1.1) can be written uniquely as

$$
\boldsymbol{u}=\stackrel{\boldsymbol{u}}{ }+\nabla \phi
$$

where $\stackrel{\boldsymbol{u}}{\in} H_{0}(\operatorname{curl} ; \Omega) \cap H\left(\operatorname{div}^{0} ; \Omega\right), \phi \in H_{0}^{1}(\Omega)$,

$$
H\left(\operatorname{div}^{0} ; \Omega\right)=\left\{\boldsymbol{v}=\left[\begin{array}{l}
v_{1} \\
v_{2}
\end{array}\right] \in\left[L_{2}(\Omega)\right]^{2}: \nabla \cdot \boldsymbol{v}=\frac{\partial v_{1}}{\partial x_{1}}+\frac{\partial v_{2}}{\partial x_{2}}=0\right\} .
$$

It is easy to see that $\phi$ satisfies

$$
\alpha(\nabla \phi, \nabla \psi)=(\boldsymbol{f}, \nabla \psi) \quad \forall \psi \in H_{0}^{1}(\Omega) .
$$

Since the Poisson problem (1.3) (when $\alpha \neq 0$ ) can be solved by many standard methods under the assumption that $f \in H(\operatorname{div} ; \Omega)$, we will focus on the divergencefree part $\stackrel{i}{\boldsymbol{u}}$, which satisfies

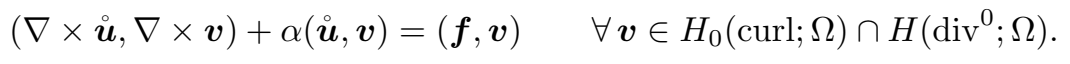

We shall refer to (1.4) as the weak form of the reduced curl-curl problem and assume in the case where $\alpha \leq 0$ that (1.4) has a unique solution (i.e. $-\alpha$ is not a Maxwell eigenvalue). Note that the strong form of the reduced curl-curl problem is given by

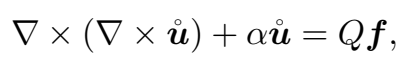

where $Q:\left[L_{2}(\Omega)\right]^{2} \longrightarrow H\left(\operatorname{div}^{0} ; \Omega\right)$ is the orthogonal projection.

Remark 1.1. The strong form (1.5) and the well-posedness of (1.4) imply immediately

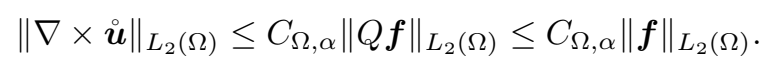

Here and below we use $C$ (with or without subscripts) to denote a generic positive constant that can take different values at different occurrences.

The advantage of working with (1.4) is that it behaves like an elliptic problem, unlike the full curl-curl problem (1.1). In particular, the solution $\stackrel{\boldsymbol{u}}{\text { of }}$ (1.4) enjoys elliptic regularity under the assumption that $\boldsymbol{f} \in L_{2}(\Omega)$, which greatly simplifies the duality argument needed for the analysis.

In [15] we developed a numerical scheme (in the case where $\alpha \leq 0$ ) for (1.4) using the Crouzeix-Raviart nonconforming $P_{1}$ vector fields [30]. In this paper we investigate an interior penalty method that avoids the difficulties involved in the construction of local bases for the Crouzeix-Raviart vector fields at the cost of a larger number of unknowns. These two methods are very closely related, which simplifies the analysis of the new scheme.

Since the solution operator of the interior penalty method converges uniformly to the solution operator of (1.4) with respect to the norm $\|\cdot\|_{L_{2}(\Omega)}$, classical spectral approximation theory [7] implies that we can also apply the interior penalty method to the following Maxwell eigenproblem:

Find $(\lambda, \stackrel{\bullet}{\boldsymbol{u}}) \in \mathbb{R} \times H_{0}(\operatorname{curl} ; \Omega) \cap H\left(\operatorname{div}^{0} ; \Omega\right)$ such that

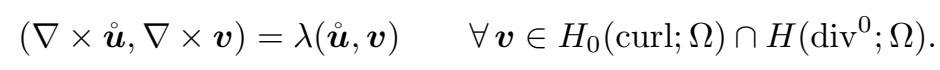


In particular, the spectral approximation based on our interior penalty method is free of spurious eigenvalues.

The rest of the paper is organized as follows. We introduce the finite element space and graded meshes in Section 2. The interior penalty method and some preliminary estimates are presented in Section 3. The convergence analysis for the source problem (1.1) is carried out in Section 4. Application to the eigenproblem (1.7) is discussed in Section 5 , followed by numerical results in Section 6 , and we end with some concluding remarks in Section 7.

2. Finite Element Spaces and Graded Meshes. In this section we will introduce discontinuous finite element spaces defined on meshes graded around the corners $c_{1}, \ldots, c_{L}$ of $\Omega$.

We assume that the triangulation $\mathcal{T}_{h}$ of $\Omega$ satisfies the following condition:

$$
h_{T} \approx h \Phi_{\mu}(T) \quad \forall T \in \mathcal{T}_{h},
$$

where $h_{T}=\operatorname{diam} T, h$ is a mesh parameter proportional to $\max _{T \in \mathcal{T}_{h}} h_{T}$, and $\mu=$ $\left(\mu_{1}, \ldots, \mu_{L}\right)$ is the vector containing the grading parameters. The constants in the equivalence (2.1) are independent of $h$ and depend in general on the minimum angle of the mesh. The weight $\Phi_{\mu}(T)$ is defined by

$$
\Phi_{\mu}(T)=\Pi_{\ell=1}^{L}\left|c_{\ell}-c_{T}\right|^{1-\mu_{\ell}},
$$

where $c_{T}$ is the center of $T$. Note that

$$
h_{T} \leq C h .
$$

The choices of the grading parameters are dictated by the singularities $[6,28,26]$ of the solution $\boldsymbol{u}$ of (1.4). In order to recover optimal convergence rates in both the energy norm and the $L_{2}$ norm (cf. [15]), we take

$$
\begin{array}{lll}
\mu_{\ell}=1 & \text { if } & \omega_{\ell} \leq \frac{\pi}{2}, \\
\mu_{\ell}<\frac{\pi}{2 \omega_{\ell}} & \text { if } & \omega_{\ell}>\frac{\pi}{2},
\end{array}
$$

where $\omega_{\ell}$ is the interior angle at the corner $c_{\ell}$. The construction of $\mathcal{T}_{h}$ satisfying (2.1) can be found for example in $[31,4,1,14]$. Note that $\mathcal{T}_{h}$ can be constructed so that it satisfies a minimum angle condition for any fixed choice of the grading parameters.

Remark 2.1. The choice of the grading parameters in (2.4) indicates that grading is needed at any corner where the angle is larger than $\pi / 2$. This is different from problems involving the Laplace operator where grading is only needed at re-entrant corners and is due to the fact that the singularities of the curl-curl operator are one order worse than the singularities of the Laplace operator.

The finite element space $V_{h}$ is defined by

$$
V_{h}=\left\{\boldsymbol{v} \in\left[L_{2}(\Omega)\right]^{2}: \boldsymbol{v}_{T}=\left.\boldsymbol{v}\right|_{T} \in\left[P_{1}(T)\right]^{2} \quad \text { and } \quad \nabla \cdot \boldsymbol{v}_{T}=0 \quad \forall T \in \mathcal{T}_{h}\right\} .
$$

For any $s>\frac{1}{2}$ there is a natural weak interpolation operator $\Pi_{T}:\left[H^{s}(T)\right]^{2} \longrightarrow$ $\left[P_{1}(T)\right]^{2}$ defined by

$$
\left(\Pi_{T} \boldsymbol{\zeta}\right)\left(m_{e_{i}}\right)=\frac{1}{\left|e_{i}\right|} \int_{e_{i}} \boldsymbol{\zeta} d s \quad \text { for } \quad i=1,2,3,
$$


where $m_{e_{i}}$ is the midpoint of the edge $e_{i}$ of $T$ and $|e|$ denotes the length of an edge $e$.

It follows immediately from (2.5), the midpoint rule and Green's theorem that

$$
\begin{aligned}
\int_{T} \nabla \times\left(\Pi_{T} \boldsymbol{\zeta}\right) d x & =\int_{T} \nabla \times \boldsymbol{\zeta} d x & & \forall \boldsymbol{\zeta} \in\left[H^{s}(T)\right]^{2}, \\
\int_{T} \nabla \cdot\left(\Pi_{T} \boldsymbol{\zeta}\right) d x & =\int_{T} \nabla \cdot \boldsymbol{\zeta} d x & & \forall \boldsymbol{\zeta} \in\left[H^{s}(T)\right]^{2} .
\end{aligned}
$$

Furthermore, given $s \in(1 / 2,2]$, we have the following interpolation error estimates [30]:

$$
\left\|\boldsymbol{\zeta}-\Pi_{T} \boldsymbol{\zeta}\right\|_{L_{2}(T)}+h_{T}^{\min (s, 1)}\left|\boldsymbol{\zeta}-\Pi_{T} \boldsymbol{\zeta}\right|_{H^{\min (s, 1)}(T)} \leq C_{T} h_{T}^{s}|\boldsymbol{\zeta}|_{H^{s}(T)}
$$

for all $\zeta \in\left[H^{s}(T)\right]^{2}$, where the positive constant $C_{T}$ depends on the minimum angle of $T$ (and also on $s$ when $s$ is close to $1 / 2$ ).

Since the solution $\boldsymbol{u}$ of the reduced curl-curl problem belongs to $\left[H^{s}(\Omega)\right]^{2}$ for some $s>\frac{1}{2}$ (cf. [40]), we can define a global interpolant of $\dot{\boldsymbol{u}}$ by

$$
\left(\Pi_{h} \stackrel{\circ}{\boldsymbol{u}}\right)_{T}=\Pi_{T} \stackrel{\circ}{u}_{T} \quad \forall T \in \mathcal{T}_{h},
$$

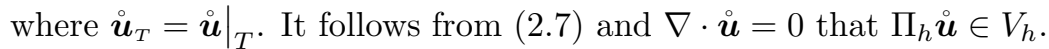

Remark 2.2. Note that $\Pi_{h} \stackrel{\boldsymbol{u}}{\text { is }}$ the same interpolation operator used in [15] and $\Pi_{h} \stackrel{\varkappa}{u}$ belongs to the space of locally divergence-free Crouzeix-Raviart nonconforming $P_{1}$ vector fields [30].

Since the vector fields in $V_{h}$ are discontinuous, their jumps across the edges of $\mathcal{T}_{h}$ play an important role in the development of interior penalty methods. We will denote by $\mathcal{E}_{h}$ (resp. $\mathcal{E}_{h}^{i}$ ) the set of the edges (resp. interior edges) of $\mathcal{T}_{h}$. Let $e \in \mathcal{E}_{h}^{i}$ be shared by the two triangles $T_{1}, T_{2} \in \mathcal{T}_{h}$ (cf. Figure 2.1) and $\boldsymbol{n}_{1}$ (resp. $\boldsymbol{n}_{2}$ ) be the unit normal of $e$ pointing towards the outside of $T_{1}$ (resp. $T_{2}$ ). We define, on $e$,

$$
\begin{aligned}
\llbracket \boldsymbol{n} \times \boldsymbol{v} \rrbracket & =\boldsymbol{n}_{1} \times\left.\boldsymbol{v}_{T_{1}}\right|_{e}+\boldsymbol{n}_{2} \times\left.\boldsymbol{v}_{T_{2}}\right|_{e}, \\
\llbracket \boldsymbol{n} \cdot \boldsymbol{v} \rrbracket & =\left.\boldsymbol{n}_{1} \cdot \boldsymbol{v}_{T_{1}}\right|_{e}+\left.\boldsymbol{n}_{2} \cdot \boldsymbol{v}_{T_{2}}\right|_{e} .
\end{aligned}
$$

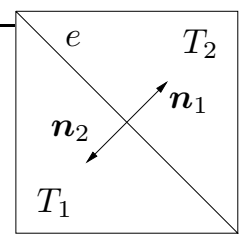

FIG. 2.1. Triangles and normals in the definitions of $\llbracket \boldsymbol{n} \times \boldsymbol{v} \rrbracket$ and $\llbracket \boldsymbol{n} \cdot \boldsymbol{v} \rrbracket$

For an edge $e$ along $\partial \Omega$, we take $\boldsymbol{n}_{e}$ to be the unit normal of $e$ pointing towards the outside of $\Omega$ and define

$$
\llbracket \boldsymbol{n} \times \boldsymbol{v} \rrbracket=\boldsymbol{n}_{e} \times\left.\boldsymbol{v}\right|_{e} .
$$

We will also denote the piecewise defined curl and div operators by $\nabla_{h} \times$ and $\nabla_{h}$, i.e.,

$$
\left.\left(\nabla_{h} \times \boldsymbol{v}\right)\right|_{T}=\nabla \times \boldsymbol{v}_{T} \quad \text { and }\left.\quad\left(\nabla_{h} \cdot \boldsymbol{v}\right)\right|_{T}=\nabla \cdot \boldsymbol{v}_{T}
$$


3. Discretization and Preliminary Error Estimates. In this section we present the interior penalty method for the reduced curl-curl problem and some preliminary estimates.

The discrete problem is to find $\dot{\boldsymbol{u}}_{h} \in V_{h}$ such that

$$
a_{h}\left(\stackrel{\varkappa}{h}_{h}, \boldsymbol{v}\right)=(\boldsymbol{f}, \boldsymbol{v}) \quad \forall \boldsymbol{v} \in V_{h},
$$

where

$$
\begin{aligned}
& a_{h}(\boldsymbol{w}, \boldsymbol{v})=\left(\nabla_{h} \times \boldsymbol{w}, \nabla_{h} \times \boldsymbol{v}\right)+\alpha(\boldsymbol{w}, \boldsymbol{v}) \\
& +\sum_{e \in \mathcal{E}_{h}} \frac{\left[\Phi_{\mu}(e)\right]^{2}}{|e|} \int_{e} \llbracket \boldsymbol{n} \times \boldsymbol{w} \rrbracket \llbracket \boldsymbol{n} \times \boldsymbol{v} \rrbracket d s \\
& +\sum_{e \in \mathcal{E}_{h}^{i}} \frac{\left[\Phi_{\mu}(e)\right]^{2}}{|e|} \int_{e} \llbracket \boldsymbol{n} \cdot \boldsymbol{w} \rrbracket \llbracket \boldsymbol{n} \cdot \boldsymbol{v} \rrbracket d s \\
& \quad+h^{-2} \sum_{e \in \mathcal{E}_{h}} \frac{1}{|e|} \int_{e}\left(\Pi_{e}^{0} \llbracket \boldsymbol{n} \times \boldsymbol{w} \rrbracket\right)\left(\Pi_{e}^{0} \llbracket \boldsymbol{n} \times \boldsymbol{v} \rrbracket\right) d s \\
& \quad+h^{-2} \sum_{e \in \mathcal{E}_{h}^{i}} \frac{1}{|e|} \int_{e}\left(\Pi_{e}^{0} \llbracket \boldsymbol{n} \cdot \boldsymbol{w} \rrbracket\right)\left(\Pi_{e}^{0} \llbracket \boldsymbol{n} \cdot \boldsymbol{v} \rrbracket\right) d s,
\end{aligned}
$$

where $\Pi_{e}^{0}$ is the orthogonal projection from $L_{2}(e)$ onto $P_{0}(e)$ (the space of constant functions on $e$ ). The weight $\Phi_{\mu}(e)$ in (3.2) is defined by

$$
\Phi_{\mu}(e)=\Pi_{\ell=1}^{L}\left|c_{\ell}-m_{e}\right|^{1-\mu_{\ell}},
$$

where $m_{e}$ is the midpoint of $e$.

Remark 3.1. The weight $\Phi_{\mu}(e)$ is closely related to the weight $\Phi_{\mu}(T)$ in (2.2). In fact, we have

$$
\Phi_{\mu}(e) \approx \Phi_{\mu}(T) \quad \text { if } \quad e \subset \partial T,
$$

where the constants in the equivalence are independent of $h$ and depend in general on the minimum angle of the mesh. The inclusion of $\Phi_{\mu}(e)$ in (3.2) is crucial for the derivation of optimal convergence rates on graded meshes.

Remark 3.2. The weak problem (1.4) for the reduced curl-curl problem can be written as

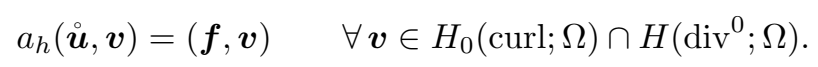

Remark 3.3. For nonconforming Crouzeix-Raviart $P_{1}$ vector fields $\boldsymbol{w}$ and $\boldsymbol{v}$, we have

$$
\begin{aligned}
& a_{h}(\boldsymbol{w}, \boldsymbol{v})=\left(\nabla_{h} \times \boldsymbol{w}, \nabla_{h} \times \boldsymbol{v}\right)+\alpha(\boldsymbol{w}, \boldsymbol{v}) \\
&+\sum_{e \in \mathcal{E}_{h}} \frac{\left[\Phi_{\mu}(e)\right]^{2}}{|e|} \int_{e} \llbracket \boldsymbol{n} \times \boldsymbol{w} \rrbracket \llbracket \boldsymbol{n} \times \boldsymbol{v} \rrbracket d s \\
& \quad+\sum_{e \in \mathcal{E}_{h}^{i}} \frac{\left[\Phi_{\mu}(e)\right]^{2}}{|e|} \int_{e} \llbracket \boldsymbol{n} \cdot \boldsymbol{w} \rrbracket \llbracket \boldsymbol{n} \cdot \boldsymbol{v} \rrbracket d s
\end{aligned}
$$


which is the variational form used in [15]. The two additional sums in (3.2) compensate for the lack of weak continuity for the vector fields in $V_{h}$.

Remark 3.4. The weak over-penalization causes the discrete system to become more ill-conditioned. For example, the condition number grows at the rate of $O\left(h^{-4}\right)$ on quasi-uniform meshes. However, there exists a simple block-diagonal preconditioner that can reduce the growth of the condition number to $O\left(h^{-2}\right)$ on quasi-uniform meshes and $O\left(h^{-2}(1+|\ln h|)\right.$ on graded meshes $[16,17]$.

Let the mesh-dependent energy norm $\|\cdot\|_{h}$ be defined by

$$
\begin{aligned}
& \|\boldsymbol{v}\|_{h}^{2}=\left\|\nabla_{h} \times \boldsymbol{v}\right\|_{L_{2}(\Omega)}^{2}+\|\boldsymbol{v}\|_{L_{2}(\Omega)}^{2} \\
& \quad+\sum_{e \in \mathcal{E}_{h}} \frac{\left[\Phi_{\mu}(e)\right]^{2}}{|e|}\|\llbracket \boldsymbol{n} \times \boldsymbol{v} \rrbracket\|_{L_{2}(e)}^{2}+\sum_{e \in \mathcal{E}_{h}^{i}} \frac{\left[\Phi_{\mu}(e)\right]^{2}}{|e|}\|\llbracket \boldsymbol{n} \cdot \boldsymbol{v} \rrbracket\|_{L_{2}(e)}^{2} \\
& \quad+h^{-2}\left(\sum_{e \in \mathcal{E}_{h}} \frac{1}{|e|}\left\|\Pi_{e}^{0} \llbracket \boldsymbol{n} \times \boldsymbol{v} \rrbracket\right\|_{L_{2}(e)}^{2}+\sum_{e \in \mathcal{E}_{h}^{i}} \frac{1}{|e|}\left\|\Pi_{e}^{0} \llbracket \boldsymbol{n} \cdot \boldsymbol{v} \rrbracket\right\|_{L_{2}(e)}^{2}\right) .
\end{aligned}
$$

Note that

$$
\|\boldsymbol{v}\|_{L_{2}(\Omega)} \leq\|\boldsymbol{v}\|_{h} \quad \forall \boldsymbol{v} \in H_{0}(\operatorname{curl} ; \Omega) \cap H\left(\operatorname{div}^{0} ; \Omega\right)+V_{h},
$$

and from (3.2) and (3.4),

$$
\left|a_{h}(\boldsymbol{w}, \boldsymbol{v})\right| \leq(|\alpha|+1)\|\boldsymbol{w}\|_{h}\|\boldsymbol{v}\|_{h} \quad \forall \boldsymbol{w}, \boldsymbol{v} \in\left[H_{0}(\operatorname{curl} ; \Omega) \cap H\left(\operatorname{div}^{0} ; \Omega\right)\right]+V_{h} .
$$

For $\alpha>0, a_{h}(\cdot, \cdot)$ is coercive with respect to $\|\cdot\|_{h}$, i.e.,

$$
a_{h}(\boldsymbol{v}, \boldsymbol{v}) \geq \min (1, \alpha)\|\boldsymbol{v}\|_{h}^{2} \quad \forall \boldsymbol{v} \in H_{0}(\operatorname{curl} ; \Omega) \cap H\left(\operatorname{div}^{0} ; \Omega\right)+V_{h} .
$$

In this case the discrete problem is well-posed and we have the following abstract error estimate.

Lemma 3.5. Let $\alpha$ be positive, $\beta=\min (1, \alpha)$, $\boldsymbol{u}$ be the solution of (1.4) and $\dot{\boldsymbol{u}}_{h}$ satisfy (3.1). It holds that

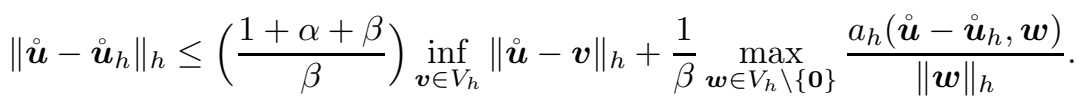

Proof. Let $\boldsymbol{v} \in V_{h}$ be arbitrary. It follows from (3.6), (3.7) and the triangle inequality that

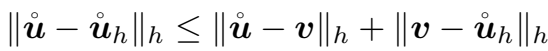

$$
\begin{aligned}
& \leq\|\stackrel{\circ}{\boldsymbol{u}}-\boldsymbol{v}\|_{h}+\frac{1}{\beta} \max _{\boldsymbol{w} \in V_{h} \backslash\{\mathbf{0}\}} \frac{a_{h}\left(\boldsymbol{v}-\stackrel{\circ}{\boldsymbol{u}}_{h}, \boldsymbol{w}\right)}{\|\boldsymbol{w}\|_{h}} \\
& \leq\left(\frac{1+\alpha+\beta}{\beta}\right)\|\stackrel{\circ}{\boldsymbol{u}}-\boldsymbol{v}\|_{h}+\frac{1}{\beta} \max _{\boldsymbol{w} \in V_{h} \backslash\{\mathbf{0}\}} \frac{a_{h}\left(\stackrel{\circ}{\boldsymbol{u}}-\stackrel{\circ}{\boldsymbol{u}}_{h}, \boldsymbol{w}\right)}{\|\boldsymbol{w}\|_{h}}
\end{aligned}
$$

which implies (3.8).

For $\alpha \leq 0$, the following Gårding (in)equality holds:

$$
a_{h}(\boldsymbol{v}, \boldsymbol{v})+(|\alpha|+1)(\boldsymbol{v}, \boldsymbol{v})=\|\boldsymbol{v}\|_{h}^{2} \quad \forall \boldsymbol{v} \in\left[H_{0}(\operatorname{curl} ; \Omega) \cap H\left(\operatorname{div}^{0} ; \Omega\right)\right]+V_{h} .
$$


In this case the discrete problem is indefinite and the following lemma provides an abstract error estimate for the scheme (3.1) under the assumption that it has a solution.

LEMMA 3.6. Let $\stackrel{\circ}{\boldsymbol{u}} \in H_{0}(\operatorname{curl} ; \Omega) \cap H\left(\operatorname{div}^{0} ; \Omega\right)$ satisfy (1.4) and $\stackrel{\mathfrak{u}}{h}_{h}$ be a solution of (3.1). It holds that

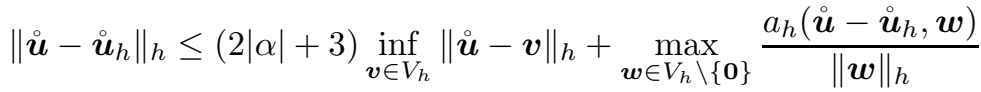

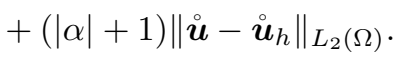

Proof. It follows from (3.5) and (3.9) that, for $\boldsymbol{v} \in V_{h} \backslash\{\mathbf{0}\}$,

$$
\begin{aligned}
\|\boldsymbol{v}\|_{h} & \leq \frac{a_{h}(\boldsymbol{v}, \boldsymbol{v})}{\|\boldsymbol{v}\|_{h}}+(|\alpha|+1) \frac{(\boldsymbol{v}, \boldsymbol{v})}{\|\boldsymbol{v}\|_{h}} \\
& \leq \max _{\boldsymbol{w} \in V_{h} \backslash\{\mathbf{0}\}} \frac{a_{h}(\boldsymbol{v}, \boldsymbol{w})}{\|\boldsymbol{w}\|_{h}}+(|\alpha|+1)\|\boldsymbol{v}\|_{L_{2}(\Omega)} .
\end{aligned}
$$

Let $\boldsymbol{v} \in V_{h}$ be arbitrary. We find, using (3.5), (3.6), (3.11) and the triangle inequality,

$$
\begin{aligned}
& \left\|\stackrel{\circ}{\boldsymbol{u}}-\stackrel{\circ}{\boldsymbol{u}}_{h}\right\|_{h} \leq\|\stackrel{\circ}{\boldsymbol{u}}-\boldsymbol{v}\|_{h}+\left\|\boldsymbol{v}-\stackrel{\circ}{\boldsymbol{u}}_{h}\right\|_{h}
\end{aligned}
$$

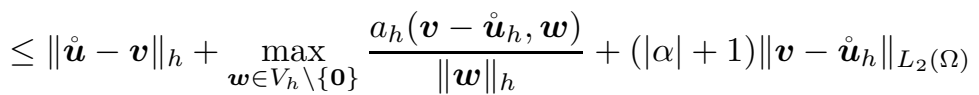

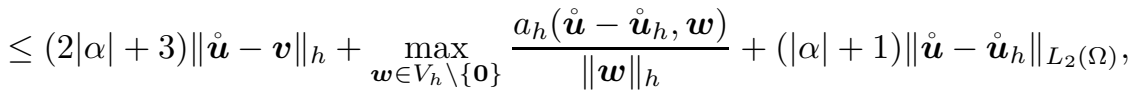

which implies (3.10).

From here on we consider $\alpha$ to be fixed and drop the dependence on $\alpha$ in our estimates.

Remark 3.7. The first term on the right-hand side of (3.8) and (3.10) measures the approximation property of $V_{h}$ with respect to the norm $\|\cdot\|_{h}$. The second term measures the consistency error. The third term on the right-hand side of (3.10) addresses the indefiniteness of the reduced curl-curl problem when $\alpha \leq 0$.

As mentioned in Remark 2.2, the interpolation operator $\Pi_{h}$ is also the one used in [15]. Therefore in our analysis we can use the following two results from that paper (cf. Lemma 5.1 and Lemma 5.2 of [15]), which were obtained using (2.4), (2.8) and a representation of $\boldsymbol{u}$ as the sum of a regular part and a singular part.

Lemma 3.8. Let $\boldsymbol{u} \in H_{0}(\operatorname{curl} ; \Omega) \cap H\left(\operatorname{div}^{0} ; \Omega\right)$ be the solution of (1.4). We have the following interpolation error estimate:

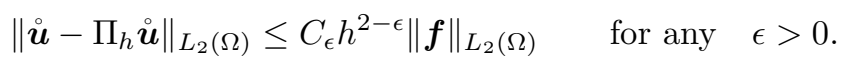

Lemma 3.9. Let $\boldsymbol{u} \in H_{0}(\operatorname{curl} ; \Omega) \cap H\left(\operatorname{div}^{0} ; \Omega\right)$ be the solution of (1.4). We have the following interpolation error estimate:

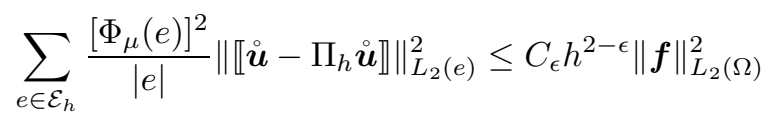




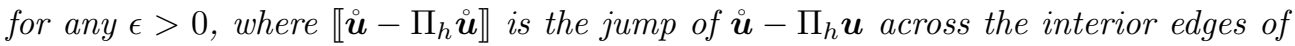

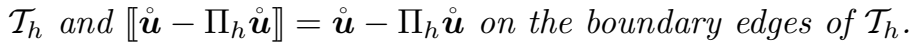

LEMMA 3.10. It holds that

$$
\sum_{e \in \mathcal{E}_{h}}|e|\left[\Phi_{\mu}(e)\right]^{-2}\left\|\eta-\bar{\eta}_{T_{e}}\right\|_{L_{2}(e)}^{2} \leq C h^{2}|\eta|_{H^{1}(\Omega)}^{2} \quad \forall \eta \in H^{1}(\Omega),
$$

where

$$
\bar{\eta}_{T_{e}}=\frac{1}{\left|T_{e}\right|} \int_{T_{e}} \eta d x
$$

is the mean of $\eta$ over $T_{e}$, one of the triangles in $\mathcal{T}_{h}$ that has $e$ as an edge.

Proof. This is the consequence of (2.1), (3.3), the trace theorem (with scaling) and a standard interpolation error estimate $[22,18]$ :

$$
\begin{aligned}
& \sum_{e \in \mathcal{E}_{h}}|e|\left[\Phi_{\mu}(e)\right]^{-2}\left\|\eta-\bar{\eta}_{T_{e}}\right\|_{L_{2}(e)}^{2} \\
& \leq C \sum_{e \in \mathcal{E}_{h}}\left[\Phi_{\mu}(T)\right]^{-2}\left(\left\|\eta-\bar{\eta}_{T_{e}}\right\|_{L_{2}\left(T_{e}\right)}^{2}+h_{T}^{2}\left|\eta-\bar{\eta}_{T_{e}}\right|_{H^{1}\left(T_{e}\right)}^{2}\right) \\
& \leq C \sum_{e \in \mathcal{E}_{h}}\left[\Phi_{\mu}(T)\right]^{-2} h_{T}^{2}|\eta|_{H^{1}\left(T_{e}\right)}^{2} \leq C h^{2}|\eta|_{H^{1}(\Omega)}^{2} .
\end{aligned}
$$

Recall that $Q$ is the $L_{2}$ orthogonal projection operator onto $H\left(\operatorname{div}^{0} ; \Omega\right)$. The following result will be useful in addressing the consistency error caused by the appearance of $Q$ in (1.5).

LEMMA 3.11. The following estimate holds:

$$
\|\boldsymbol{v}-Q \boldsymbol{v}\|_{L_{2}(\Omega)} \leq C h\|\boldsymbol{v}\|_{h} \quad \forall \boldsymbol{v} \in\left[H_{0}(\operatorname{curl} ; \Omega) \cap H\left(\operatorname{div}^{0} ; \Omega\right)\right]+V_{h} .
$$

Proof. Let $\boldsymbol{v} \in\left[H_{0}(\operatorname{curl} ; \Omega) \cap H\left(\operatorname{div}^{0} ; \Omega\right)\right]+V_{h}$ be arbitrary. Since $\boldsymbol{v}-Q \boldsymbol{v}$ belongs to $\nabla H_{0}^{1}(\Omega)$, the orthogonal complement of $H\left(\operatorname{div}^{0} ; \Omega\right)$ in $\left[L_{2}(\Omega)\right]^{2}$, we have, by duality,

$$
\|\boldsymbol{v}-Q \boldsymbol{v}\|_{L_{2}(\Omega)}=\sup _{\eta \in H_{0}^{1}(\Omega) \backslash\{0\}} \frac{(\boldsymbol{v}-Q \boldsymbol{v}, \nabla \eta)}{\|\nabla \eta\|_{L_{2}(\Omega)}}=\sup _{\eta \in H_{0}^{1}(\Omega) \backslash\{0\}} \frac{(\boldsymbol{v}, \nabla \eta)}{\|\nabla \eta\|_{L_{2}(\Omega)}} .
$$

Let $\eta \in H_{0}^{1}(\Omega)$ be arbitrary. Since $\nabla \cdot \boldsymbol{v}=0$ on each triangle $T \in \mathcal{T}_{h}$, we find using integration by parts and the fact that $\left.\eta\right|_{\partial \Omega}=0$,

$$
(\boldsymbol{v}, \nabla \eta)=\sum_{e \in \mathcal{E}_{h}^{i}} \int_{e}\left(\eta-\bar{\eta}_{T_{e}}\right) \llbracket \boldsymbol{n} \cdot \boldsymbol{v} \rrbracket d s+\sum_{e \in \mathcal{E}_{h}^{i}} \int_{e} \bar{\eta}_{T_{e}}\left(\Pi_{e}^{0} \llbracket \boldsymbol{n} \cdot \boldsymbol{v} \rrbracket\right) d s=S_{1}+S_{2},
$$

where $\bar{\eta}_{T_{e}}$ is defined in (3.14).

By the Cauchy-Schwarz inequality, (3.4) and Lemma 3.10, we have

$$
\begin{aligned}
S_{1} & \leq\left[\sum_{e \in \mathcal{E}_{h}^{i}}|e|\left[\Phi_{\mu}(e)\right]^{-2}\left\|\eta-\bar{\eta}_{T_{e}}\right\|_{L_{2}(e)}^{2}\right]^{1 / 2}\left[\sum_{e \in \mathcal{E}_{h}^{i}} \frac{\left[\Phi_{\mu}(e)\right]^{2}}{|e|}\|\llbracket \boldsymbol{n} \cdot \boldsymbol{v} \rrbracket\|_{L_{2}(e)}^{2}\right]^{1 / 2} \\
& \leq C h\|\nabla \eta\|_{L_{2}(\Omega)}\|\boldsymbol{v}\|_{h} .
\end{aligned}
$$


On the other hand, from (3.4), the Cauchy-Schwarz inequality, and a PoincaréFriedrichs inequality, we find

$$
\begin{aligned}
S_{2} & \leq \sum_{e \in \mathcal{E}_{h}^{i}}\left(|e|^{1 / 2}\left\|\bar{\eta}_{T_{e}}\right\|_{L_{2}(e)}\right)\left(|e|^{-1 / 2}\left\|\Pi_{e}^{0} \llbracket \boldsymbol{n} \cdot \boldsymbol{v} \rrbracket\right\|_{L_{2}(e)}\right) \\
& \leq C h\left[\sum_{e \in \mathcal{E}_{h}^{i}}\left\|\bar{\eta}_{T_{e}}\right\|_{L_{2}\left(T_{e}\right)}^{2}\right]^{1 / 2}\left[h^{-2} \sum_{e \in \mathcal{E}_{h}^{i}} \frac{1}{|e|}\left\|\Pi_{e}^{0} \llbracket \boldsymbol{n} \cdot \boldsymbol{v} \rrbracket\right\|_{L_{2}(e)}^{2}\right]^{1 / 2} \\
& \leq C h\|\eta\|_{L_{2}(\Omega)}\|\boldsymbol{v}\|_{h} \leq C h\|\nabla \eta\|_{L_{2}(\Omega)}\|\boldsymbol{v}\|_{h} .
\end{aligned}
$$

Here we have also used the simple fact that, if $e$ is an edge of a triangle $T$, then

$$
|e|\|q\|_{L_{2}(e)}^{2} \leq C_{T}\|q\|_{L_{2}(T)}^{2} \text { for any constant function } q,
$$

where the positive constant $C_{T}$ depends only on the shape regularity of $T$.

The estimate (3.15) follows from (3.16)-(3.19).

4. Convergence Analysis. We begin with three lemmas that provide estimates to the three terms on the right-hand side of (3.8) and (3.10).

Lemma 4.1. Let $\stackrel{\boldsymbol{u}}{\in} H_{0}(\operatorname{curl} ; \Omega) \cap H\left(\operatorname{div}^{0} ; \Omega\right)$ be the solution of (1.4). It holds that

$$
\inf _{\boldsymbol{v} \in V_{h}}\|\stackrel{\circ}{\boldsymbol{u}}-\boldsymbol{v}\|_{h} \leq\left\|\stackrel{\circ}{\boldsymbol{u}}-\Pi_{h} \stackrel{\circ}{\boldsymbol{o}}\right\|_{h} \leq C_{\epsilon} h^{1-\epsilon}\|\boldsymbol{f}\|_{L_{2}(\Omega)}
$$

for any $\epsilon>0$.

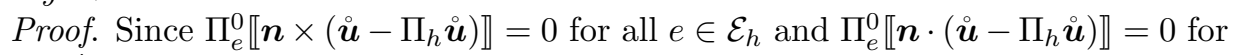
all $e \in \mathcal{E}_{h}^{i}$, we have

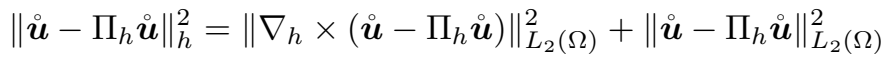

$$
\begin{aligned}
& +\sum_{e \in \mathcal{E}_{h}} \frac{\left[\Phi_{\mu}(e)\right]^{2}}{|e|}\left\|\llbracket \boldsymbol{n} \times\left(\stackrel{\circ}{\boldsymbol{u}}-\Pi_{h} \stackrel{\circ}{\boldsymbol{u}}\right) \rrbracket\right\|_{L_{2}(e)}^{2} \\
& +\sum_{e \in \mathcal{E}_{h}^{i}} \frac{\left[\Phi_{\mu}(e)\right]^{2}}{|e|}\left\|\llbracket \boldsymbol{n} \cdot\left(\stackrel{\mathfrak{u}}{ }-\Pi_{h} \stackrel{\leftrightarrow}{u}\right) \rrbracket\right\|_{L_{2}(e)}^{2} .
\end{aligned}
$$

The second term on the right-hand side of (4.2) has been estimated in Lemma 3.8, and the third and fourth terms can be estimated using Lemma 3.9. Therefore it only remains to estimate the first term.

Observe that (2.6) implies

$$
\nabla_{h} \times\left(\Pi_{h} \stackrel{\leftrightarrow}{u}\right)=\Pi_{h}^{0}(\nabla \times \stackrel{\mathfrak{u}}{)},
$$

where $\Pi_{h}^{0}$ is the orthogonal projection from $L_{2}(\Omega)$ onto the space of piecewise constant functions with respect to $\mathcal{T}_{h}$. It then follows from (1.6), (2.3), (4.3) and a standard interpolation error estimate $[22,18]$ that

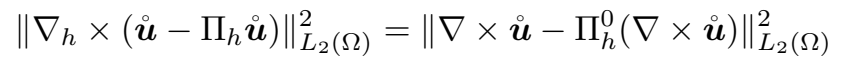

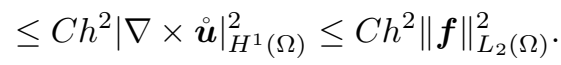

The estimate (4.1) follows from (4.2), (4.4) and Lemmas 3.8-3.9. 
Lemma 4.2. Let $\dot{\boldsymbol{u}} \in H_{0}(\operatorname{curl} ; \Omega) \cap H\left(\operatorname{div}^{0} ; \Omega\right)$ be the solution of (1.4) and $\dot{\boldsymbol{u}}_{h} \in V_{h}$ satisfy (3.1). It holds that

$$
\max _{\boldsymbol{w} \in V_{h} \backslash\{\mathbf{0}\}} \frac{a_{h}\left(\stackrel{\circ}{\boldsymbol{u}}-{\left.\stackrel{\circ}{\boldsymbol{u}_{h}}, \boldsymbol{w}\right)}\right.}{\|\boldsymbol{w}\|_{h}} \leq C h\|\boldsymbol{f}\|_{L_{2}(\Omega)} .
$$

Proof. Let $\boldsymbol{w} \in V_{h}$ be arbitrary. Using integration by parts, the discrete problem (3.1), the strong form of the reduced curl-curl problem (1.5), and the fact that $\nabla_{h} \cdot \boldsymbol{w}=$ 0 , we find

$$
\begin{aligned}
& a_{h}\left(\stackrel{\circ}{\boldsymbol{u}}-\stackrel{\circ}{\boldsymbol{u}}_{h}, \boldsymbol{w}\right)=a_{h}(\stackrel{\circ}{\boldsymbol{u}}, \boldsymbol{w})-(\boldsymbol{f}, \boldsymbol{w}) \\
& =(Q \boldsymbol{f}-\boldsymbol{f}, \boldsymbol{w})+\sum_{T \in \mathcal{T}_{h}} \int_{\partial T}(\nabla \times \dot{u})\left(\boldsymbol{n}_{T} \times \boldsymbol{w}_{T}\right) d s,
\end{aligned}
$$

where $w_{T}=\left.w\right|_{T}$ and $\boldsymbol{n}_{T}$ is the unit outer normal along $\partial T$. We see from (4.6) that there are two sources for the inconsistency of the scheme defined by (3.1), namely the projection $Q$ that appears in (1.5) and the discontinuity of the vector fields in $V_{h}$.

The equation (4.6) can be rewritten as

$$
\begin{aligned}
& a_{h}\left(\stackrel{\circ}{\boldsymbol{u}}-\stackrel{\circ}{\boldsymbol{u}}_{h}, \boldsymbol{w}\right)=(\boldsymbol{f}, Q \boldsymbol{w}-\boldsymbol{w})+\sum_{e \in \mathcal{E}_{h}} \int_{e}(\nabla \times \stackrel{\circ}{e}) \llbracket \boldsymbol{n} \times \boldsymbol{w} \rrbracket d s \\
& =(\boldsymbol{f}, Q \boldsymbol{w}-\boldsymbol{w})+\sum_{e \in \mathcal{E}_{h}} \int_{e}\left(\nabla \times \stackrel{\circ}{ }-{\overline{(\nabla \times \mathfrak{u}})_{T_{e}}}_{)} \llbracket \boldsymbol{n} \times \boldsymbol{w} \rrbracket d s\right. \\
& +\sum_{e \in \mathcal{E}_{h}} \int_{e}{\overline{(\nabla \times \dot{u})_{T}}}_{T_{e}}\left(\Pi_{e}^{0} \llbracket \boldsymbol{n} \times \boldsymbol{w} \rrbracket\right) d s
\end{aligned}
$$

where $\overline{(\nabla \times \mathfrak{u})}_{T_{e}}$ is the mean of $\nabla \times \dot{\boldsymbol{u}}$ on one of the triangles $\mathcal{T}_{e} \in \mathcal{T}_{h}$ that has $e$ as an edge. The first two terms on the right-hand side of (4.7) satisfy the estimate

$$
(\boldsymbol{f}, Q \boldsymbol{w}-\boldsymbol{w})+\sum_{e \in \mathcal{E}_{h}} \int_{e}\left(\nabla \times \stackrel{\circ}{ }-{\overline{(\nabla \times \dot{\boldsymbol{u}}})_{T_{e}}}\right) \llbracket \boldsymbol{n} \times \boldsymbol{w} \rrbracket d s \leq C h\|\boldsymbol{f}\|_{L_{2}(\Omega)}\|\boldsymbol{w}\|_{h} .
$$

The derivation of (4.8), which is based on Lemma 3.10, Lemma 3.11 and (1.6), can be found in the proof of Lemma 6.2 in [15].

Using the Cauchy-Schwarz inequality, (1.6), (3.4) and (3.20), the third term on the right-hand side of (4.7) can be estimated as follows:

$$
\begin{aligned}
\sum_{e \in \mathcal{E}_{h}} \int_{e} & {\overline{(\nabla \times \mathfrak{u})_{T_{e}}}}_{\left(\Pi_{e}^{0} \llbracket \boldsymbol{n} \times \boldsymbol{w} \rrbracket\right) d s} \\
& \leq \sum_{e \in \mathcal{E}_{h}}\left(|e|^{1 / 2}\left\|\overline{(\nabla \times \boldsymbol{i})_{T_{e}}}\right\|_{L_{2}(e)}\right)\left(|e|^{-1 / 2}\left\|\Pi_{e}^{0} \llbracket \boldsymbol{n} \times \boldsymbol{w} \rrbracket\right\|_{L_{2}(e)}\right) \\
& \leq C h\left(\sum_{e \in \mathcal{E}_{h}}\left\|\overline{(\nabla \times \mathfrak{u})_{T_{e}}}\right\|_{L_{2}\left(T_{e}\right)}^{2}\right)^{1 / 2}\left(h^{-2} \sum_{e \in \mathcal{E}_{h}} \frac{1}{|e|}\left\|\Pi_{e}^{0} \llbracket \boldsymbol{n} \times \boldsymbol{w} \rrbracket\right\|_{L_{2}(e)}^{2}\right)^{1 / 2} \\
& \leq C h\|\nabla \times \mathfrak{u}\|_{L_{2}(\Omega)}\|\boldsymbol{w}\|_{h} \leq C h\|\boldsymbol{f}\|_{L_{2}(\Omega)}\|\boldsymbol{w}\|_{h} .
\end{aligned}
$$

The estimate (4.5) follows from (4.7)-(4.9). 
Lemma 4.3. Let $\boldsymbol{u} \in H_{0}(\operatorname{curl} ; \Omega) \cap H\left(\operatorname{div}^{0} ; \Omega\right)$ be the solution of (1.4) and $\dot{\boldsymbol{u}}_{h} \in V_{h}$ satisfy (3.1). It holds that

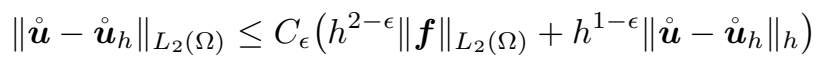

for any $\epsilon>0$.

Proof. We use a duality argument. Let $\stackrel{\circ}{z} \in H_{0}(\operatorname{curl} ; \Omega) \cap H\left(\operatorname{div}^{0} ; \Omega\right)$ satisfy the reduced curl-curl problem

$$
a_{h}(\boldsymbol{v}, \stackrel{\circ}{\boldsymbol{z}})=\left(\boldsymbol{v}, \stackrel{\circ}{\boldsymbol{u}}-\stackrel{\circ}{\boldsymbol{u}}_{h}\right) \quad \forall \boldsymbol{v} \in H_{0}(\operatorname{curl} ; \Omega) \cap H\left(\operatorname{div}^{0} ; \Omega\right) .
$$

The strong form of (4.11) is

$$
\nabla \times\left(\nabla \times \stackrel{\check{z}}{)}+\alpha \dot{\boldsymbol{z}}=Q\left(\stackrel{\circ}{\boldsymbol{u}}-\dot{\boldsymbol{u}}_{h}\right),\right.
$$

and we have the following analog of (1.6):

$$
\|\nabla \times \stackrel{\gtrless}{z}\|_{H^{1}(\Omega)} \leq C_{\Omega}\left\|\stackrel{\circ}{\boldsymbol{u}}-\stackrel{\circ}{\boldsymbol{u}}_{h}\right\|_{L_{2}(\Omega)} .
$$

It follows from (4.12) and integration by parts that

$$
a_{h}\left(\stackrel{\circ}{\boldsymbol{u}}_{h}, \stackrel{\check{z}}{)}=\left(\stackrel{\circ}{\boldsymbol{u}}_{h}, Q\left(\stackrel{\circ}{\boldsymbol{u}}-\stackrel{\circ}{\boldsymbol{u}}_{h}\right)\right)+\sum_{e \in \mathcal{E}_{h}} \int_{e}\left(\nabla \times \stackrel{\mathrm{z}}{)} \llbracket \boldsymbol{n} \times \stackrel{\circ}{\boldsymbol{u}}_{h} \rrbracket d s .\right.\right.
$$

From (4.11) and (4.14), we have

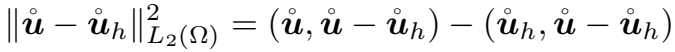

$$
\begin{aligned}
& =a_{h}\left(\stackrel{\circ}{\boldsymbol{u}}-\stackrel{\circ}{\boldsymbol{u}}_{h}, \stackrel{\circ}{\boldsymbol{z}}\right)+a_{h}\left(\stackrel{\circ}{\boldsymbol{u}}_{h}, \stackrel{\circ}{\boldsymbol{z}}\right)-\left(\stackrel{\circ}{\boldsymbol{u}}_{h}, \stackrel{\circ}{\boldsymbol{u}}-\dot{\circ}_{h}\right)
\end{aligned}
$$

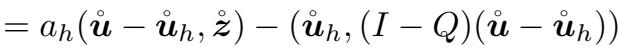

$$
\begin{aligned}
& +\sum_{e \in \mathcal{E}_{h}} \int_{e}(\nabla \times \stackrel{z}{*}) \llbracket \boldsymbol{n} \times \stackrel{\mathfrak{u}}{h}_{h} \rrbracket d s .
\end{aligned}
$$

We will estimate the three terms on the right-hand side of (4.15) separately.

Using (4.7) and the fact that $\llbracket \boldsymbol{n} \times\left(\Pi_{h} \dot{\boldsymbol{z}}\right) \rrbracket$ vanishes at the midpoints of all $e \in \mathcal{E}_{h}$, we can rewrite the first term as

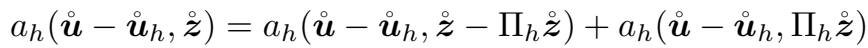

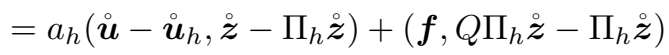

$$
\begin{aligned}
& +\sum_{e \in \mathcal{E}_{h}} \int_{e}\left(\nabla \times \stackrel{\mathfrak{u}}{ }-\overline{(\nabla \times \stackrel{\boldsymbol{u}}{ })}_{T_{e}}\right) \llbracket \boldsymbol{n} \times\left(\Pi_{h} \stackrel{\boldsymbol{z}}{)}\right) \rrbracket d s,
\end{aligned}
$$

where $\overline{(\nabla \times \mathfrak{u})}_{T_{e}}$ is the mean of $\nabla \times \dot{\boldsymbol{u}}$ on one of the triangles $\mathcal{T}_{e} \in \mathcal{T}_{h}$ that has $e$ as an edge. Using the expression (4.16), the following estimate was obtained in the proof of Lemma 6.5 of [15]:

$$
a_{h}\left(\stackrel{\circ}{\boldsymbol{u}}-\stackrel{\circ}{\boldsymbol{u}}_{h}, \stackrel{\circ}{\boldsymbol{z}}\right) \leq C_{\epsilon}\left(h^{2-\epsilon}\|\boldsymbol{f}\|_{L_{2}(\Omega)}+h^{1-\epsilon}\left\|\stackrel{\circ}{\boldsymbol{u}}-\stackrel{\circ}{u}_{h}\right\|_{h}\right)\left\|\stackrel{\circ}{\boldsymbol{u}}-\stackrel{\circ}{\boldsymbol{u}}_{h}\right\|_{L_{2}(\Omega)} .
$$

Since $(I-Q) \dot{\boldsymbol{u}}=\mathbf{0}$, the second term on the right-hand side of (4.15) can be estimated by Lemma 3.11:

$$
-\left(\stackrel{\circ}{\boldsymbol{u}}_{h},(I-Q)\left(\stackrel{\circ}{\boldsymbol{u}}-\stackrel{\circ}{\boldsymbol{u}}_{h}\right)\right)=\left(\stackrel{\circ}{\boldsymbol{u}}-\stackrel{\circ}{\boldsymbol{u}}_{h},(I-Q)\left(\stackrel{\circ}{\boldsymbol{u}}-\stackrel{\circ}{\boldsymbol{u}}_{h}\right)\right)
$$




$$
\leq C\left\|\stackrel{\circ}{ }-\stackrel{\circ}{u}_{h}\right\|_{L_{2}(\Omega)}\left(h\left\|\stackrel{\circ}{ }-\stackrel{\circ}{u}_{h}\right\|_{h}\right) .
$$

Finally we estimate the third term on the right-hand side of (4.15). We have

$$
\begin{aligned}
& \sum_{e \in \mathcal{E}_{h}} \int_{e}(\nabla \times \stackrel{2}{\boldsymbol{z}}) \llbracket \boldsymbol{n} \times \stackrel{\circ}{\boldsymbol{u}}_{h} \rrbracket d s=\sum_{e \in \mathcal{E}_{h}} \int_{e}\left(\nabla \times \stackrel{\boldsymbol{z}}{ }-\overline{(\nabla \times \stackrel{\boldsymbol{z}}{ })}_{T_{e}}\right) \llbracket \boldsymbol{n} \times \dot{\boldsymbol{u}}_{h} \rrbracket d s \\
& +\sum_{e \in \mathcal{E}_{h}} \int_{e} \overline{(\nabla \times \stackrel{z}{ })}_{T_{e}}\left(\Pi_{e}^{0} \llbracket \boldsymbol{n} \times \stackrel{\circ}{u}_{h} \rrbracket\right) d s,
\end{aligned}
$$

where $\overline{(\nabla \times \mathscr{z})}_{T_{e}}$ is the mean of $\nabla \times \dot{z}$ on a triangle $T_{e} \in \mathcal{T}_{h}$ that has $e$ as an edge. The following estimate can be found in the proof of Lemma 6.5 of [15]:

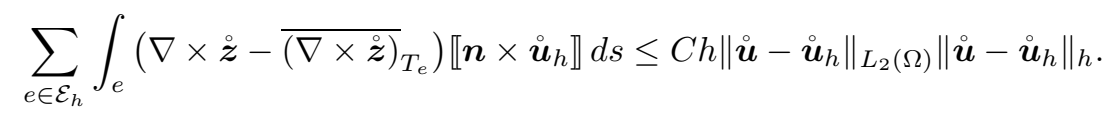

On the other hand, we obtain by the Cauchy-Schwarz inequality, (3.4), (3.20) and (4.13),

$$
\begin{aligned}
& \sum_{e \in \mathcal{E}_{h}} \int_{e} \overline{(\nabla \times \stackrel{2}{)}}_{T_{e}}\left(\Pi_{e}^{0} \llbracket \boldsymbol{n} \times \stackrel{\circ}{\boldsymbol{u}}_{h} \rrbracket\right) d s \\
& =\sum_{e \in \mathcal{E}_{h}} \int_{e} \overline{(\nabla \times \stackrel{2}{)}}_{T_{e}}\left(\Pi_{e}^{0} \llbracket \boldsymbol{n} \times\left(\stackrel{\circ}{\boldsymbol{u}}_{h}-\grave{\boldsymbol{u}}\right) \rrbracket\right) d s
\end{aligned}
$$

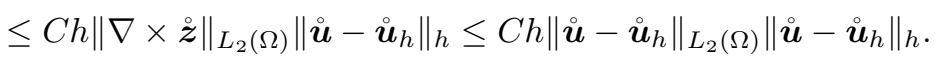

The estimate (4.10) follows from (4.15) and (4.17)-(4.21).

In the case where $\alpha>0$, the following theorem is an immediate consequence of Lemma 3.5 and Lemmas 4.1-4.3.

THEOREM 4.4. Let $\alpha$ be positive. The following estimates hold for the solution $\stackrel{\circ}{\boldsymbol{u}}_{h}$ of $(3.1)$ :

$$
\begin{aligned}
& \left\|\stackrel{\circ}{-}-\stackrel{\circ}{u}_{h}\right\|_{h} \leq C_{\epsilon} h^{1-\epsilon}\|\boldsymbol{f}\|_{L_{2}(\Omega)} \quad \text { for any } \epsilon>0,
\end{aligned}
$$

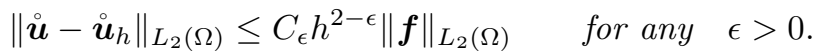

In the case where $\alpha \leq 0$, we have the following convergence theorem for the scheme (3.1).

TheOREM 4.5. Assume that $-\alpha \geq 0$ is not a Maxwell eigenvalue. There exists a positive number $h_{*}$ such that the discrete problem (3.1) is uniquely solvable for all $h \leq h_{*}$, in which case the following discretization error estimates are valid:

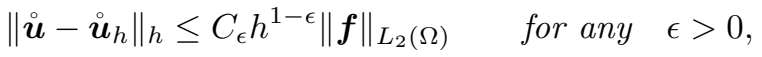

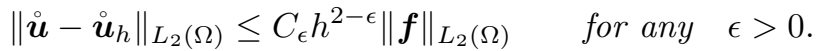

Proof. We follow the approach of Schatz [44] for indefinite problems. Assuming $\stackrel{\circ}{\boldsymbol{u}}_{h}$ satisfies (3.1), it follows from (3.10) and Lemmas 4.1-4.3 that

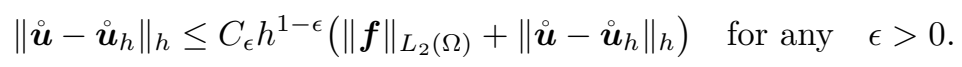


By choosing an $\epsilon_{*}>0$, we deduce from (4.26) that, for $h \leq h_{*}=1 /\left(2 C_{\epsilon_{*}}\right)^{1 /\left(1-\epsilon_{*}\right)}$,

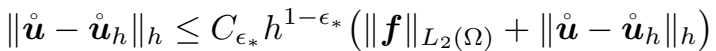

$$
\begin{aligned}
& \leq C_{\epsilon_{*}} h^{1-\epsilon_{*}}\|\boldsymbol{f}\|_{L_{2}(\Omega)}+C_{\epsilon_{*}} h_{*}^{1-\epsilon_{*}}\left\|\stackrel{i}{\boldsymbol{u}}-\dot{\boldsymbol{u}}_{h}\right\|_{h} \\
& \leq C_{\epsilon_{*}} h^{1-\epsilon_{*}}\|\boldsymbol{f}\|_{L_{2}(\Omega)}+\frac{1}{2}\left\|\stackrel{\circ}{\boldsymbol{u}}-\stackrel{\circ}{u}_{h}\right\|_{h},
\end{aligned}
$$

and hence

$$
\left\|\stackrel{\circ}{\boldsymbol{u}}-\stackrel{\circ}{\boldsymbol{u}}_{h}\right\|_{h} \leq 2 C_{\epsilon_{*}} h^{1-\epsilon_{*}}\|\boldsymbol{f}\|_{L_{2}(\Omega)} .
$$

Therefore, any solution $\stackrel{\check{z}}{h}_{h} \in V_{h}$ of the homogeneous discrete problem

$$
a_{h}\left(\stackrel{\circ}{h}_{h}, \boldsymbol{v}\right)=0 \quad \forall \boldsymbol{v} \in V_{h},
$$

which corresponds to the special case where $\boldsymbol{f}=\mathbf{0}=\stackrel{2}{\boldsymbol{z}}$, will satisfy the following special case of (4.27):

$$
\left\|\dot{z}_{h}\right\|_{h} \leq 0 .
$$

Hence the only solution of (4.28) is the trivial solution and the discrete problem (3.1) is uniquely solvable for $h \leq h_{*}$.

The energy error estimate (4.24) now follows (4.27), and the $L_{2}$ error estimate (4.25) follows from Lemma 4.3 and (4.24).

5. Application to the Maxwell Eigenproblem. Given any $\boldsymbol{f} \in\left[L_{2}(\Omega)\right]^{2}$, we define $T \boldsymbol{f} \in H_{0}(\operatorname{curl} ; \Omega) \cap H\left(\operatorname{div}^{0} ; \Omega\right)$ by the condition that

$$
(\nabla \times(T \boldsymbol{f}), \nabla \times \boldsymbol{v})+(T \boldsymbol{f}, \boldsymbol{v})=(\boldsymbol{f}, \boldsymbol{v}) \quad \forall \boldsymbol{v} \in H_{0}(\operatorname{curl} ; \Omega) \cap H\left(\operatorname{div}^{0} ; \Omega\right) .
$$

Clearly $T$ is a bounded linear operator from $\left[L_{2}(\Omega)\right]^{2}$ into $H_{0}(\operatorname{curl} ; \Omega) \cap H\left(\operatorname{div}^{0} ; \Omega\right)$. Since $H_{0}(\operatorname{curl} ; \Omega) \cap H\left(\operatorname{div}^{0} ; \Omega\right)$ is a compact subspace of $\left[L_{2}(\Omega)\right]^{2}$ (cf. [23]), the operator $T:\left[L_{2}(\Omega)\right]^{2} \longrightarrow\left[L_{2}(\Omega)\right]^{2}$ is symmetric, positive and compact. Moreover, $(\lambda, \boldsymbol{u})$ satisfy the Maxwell eigenproblem (1.7) if and only if

$$
T \stackrel{\circ}{\boldsymbol{u}}=\frac{1}{1+\lambda} \stackrel{\bullet}{\boldsymbol{u}} .
$$

Similarly, let $T_{h} \boldsymbol{f} \in V_{h}$ be defined by the condition

$$
a_{h, 1}\left(T_{h} \boldsymbol{f}, \boldsymbol{v}\right)=(\boldsymbol{f}, \boldsymbol{v}) \quad \forall \boldsymbol{v} \in V_{h},
$$

where $a_{h, 1}(\cdot, \cdot)$ is the bilinear form $a_{h}(\cdot, \cdot)$ in $(3.2)$ with $\alpha=1$, then

$$
T_{h} \stackrel{\circ}{h}_{h}=\frac{1}{1+\lambda_{h}} \stackrel{\circ}{h}_{h}
$$

is equivalent to

$$
a_{h, 0}\left(\stackrel{\circ}{\boldsymbol{u}}_{h}, \boldsymbol{v}\right)=\lambda_{h}\left(\stackrel{\circ}{\boldsymbol{u}}_{h}, \boldsymbol{v}\right) \quad \forall \boldsymbol{v} \in V_{h},
$$

where $a_{h, 0}(\cdot, \cdot)$ is the bilinear form $a_{h}(\cdot, \cdot)$ in $(3.2)$ with $\alpha=0$.

From Theorem 4.4 we have

$$
\left\|\left(T-T_{h}\right) \boldsymbol{f}\right\|_{L_{2}(\Omega)} \leq C_{\epsilon} h^{2-\epsilon}\|\boldsymbol{f}\|_{L_{2}(\Omega)}
$$


for all $\boldsymbol{f} \in\left[L_{2}(\Omega)\right]^{2}$ and

$$
\left\|\left(T-T_{h}\right) \boldsymbol{f}\right\|_{h} \leq C_{\epsilon} h^{1-\epsilon}\|\boldsymbol{f}\|_{L_{2}(\Omega)} \leq C_{\epsilon} h^{1-\epsilon}\|\boldsymbol{f}\|_{h}
$$

for all $\boldsymbol{f} \in H_{0}(\operatorname{curl} ; \Omega) \cap H\left(\operatorname{div}^{0} ; \Omega\right)+V_{h}$. Thus the symmetric finite rank operator $T_{h}$ converges uniformly to the symmetric positive compact operator $T$ as $h \downarrow 0$, which implies that the classical theory of spectral approximation [39, 7] can be applied to the approximation of the eigenvalues and eigenfunctions of $T$ by the eigenvalues and eigenfunctions of $T_{h}$. Hence we have the following result through the connections between (1.7) and (5.2) and between (5.5) and (5.4).

Theorem 5.1. Let $0 \leq \lambda_{1} \leq \lambda_{2} \leq \ldots$ be the eigenvalues of (1.7), $\lambda=\lambda_{j}=$ $\lambda_{j+1}=\cdots=\lambda_{j+m-1}$ be an eigenvalue with multiplicity $m$, and $V_{\lambda} \subset H_{0}(\operatorname{curl} ; \Omega) \cap$ $H\left(\operatorname{div}^{0} ; \Omega\right)$ be the corresponding $m$ dimensional eigenspace. Let $\lambda_{h, 1} \leq \lambda_{h, 2} \leq \ldots$ be the eigenvalues of (5.5). Then, as $h \downarrow 0$, we have

$$
\left|\lambda_{h, \ell}-\lambda\right| \leq C_{\lambda, \epsilon} h^{2-\epsilon} \quad \ell=j, \ldots, j+m-1 .
$$

Furthermore, if $V_{h, \lambda} \subset V_{h}$ is the space spanned by the eigenfunctions corresponding to $\lambda_{h, j}, \ldots, \lambda_{h, j+m-1}$, then the gap between $V_{\lambda}$ and $V_{h, \lambda}$ goes to zero at the rate of $C_{\epsilon} h^{2-\epsilon}$ in the $L_{2}$ norm and at the rate of $C_{\epsilon} h^{1-\epsilon}$ in the norm $\|\cdot\|_{h}$.

Proof. Let $E_{\lambda}$ (resp. $E_{h, \lambda}$ ) be the $L_{2}$ orthogonal projection onto $V_{\lambda}$ (resp. $V_{h, \lambda}$ ). It follows from (5.6)-(5.7) and the classical theory of spectral approximation of compact operators $[39,7]$ that

$$
\left\|\left(E_{\lambda}-E_{h, \lambda}\right) \boldsymbol{w}\right\|_{L_{2}(\Omega)} \leq C_{\epsilon} h^{2-\epsilon}\|\boldsymbol{w}\|_{L_{2}(\Omega)}
$$

for all $\boldsymbol{w} \in\left[L_{2}(\Omega)\right]^{2}$ and

$$
\left\|\left(E_{\lambda}-E_{h, \lambda}\right) \boldsymbol{w}\right\|_{h} \leq C_{\epsilon} h^{1-\epsilon}\|\boldsymbol{w}\|_{h}
$$

for all $\boldsymbol{w} \in H_{0}(\operatorname{curl} ; \Omega) \cap H\left(\operatorname{div}^{0} ; \Omega\right)+V_{h}$.

Let $\boldsymbol{x} \in V_{h, \lambda}$ be a unit eigenfunction of $\lambda_{h, \ell}$ for some $\ell$ between $j$ and $j+m-1$. Then we have

$$
\left(T_{h} \boldsymbol{x}, \boldsymbol{x}\right)=\mu_{h, \ell} \quad \text { and } \quad\|\boldsymbol{x}\|_{L_{2}(\Omega)}=1,
$$

where (cf. (5.4))

$$
\mu_{h, \ell}=\left(1+\lambda_{h, \ell}\right)^{-1} .
$$

Let $\hat{\boldsymbol{x}}=E_{\lambda} \boldsymbol{x}$ and $\hat{\boldsymbol{y}}=\hat{\boldsymbol{x}}-\boldsymbol{x}$. Then $\hat{\boldsymbol{x}}$ and $\hat{\boldsymbol{y}}$ are orthogonal with respect to both the $L_{2}$ inner product and the inner product $(T \cdot, \cdot)$, and the estimate (5.9) implies that

$$
\|\hat{\boldsymbol{y}}\|_{L_{2}(\Omega)}=\left\|\left(E_{\lambda}-E_{h, \lambda}\right) \boldsymbol{x}\right\|_{L_{2}(\Omega)} \leq C_{\epsilon} h^{2-\epsilon} .
$$

In particular, it follows from Pythagoras' theorem with respect to the $L_{2}$ inner product that

$$
1-\|\hat{\boldsymbol{x}}\|_{L_{2}(\Omega)}^{2}=\|\boldsymbol{x}\|_{L_{2}(\Omega)}^{2}-\|\hat{\boldsymbol{x}}\|_{L_{2}(\Omega)}^{2}=\|\hat{\boldsymbol{y}}\|_{L_{2}(\Omega)}^{2} \leq C_{\epsilon} h^{4-\epsilon} .
$$

Let $\hat{\boldsymbol{e}}=\hat{\boldsymbol{x}} /\|\hat{\boldsymbol{x}}\|_{L_{2}(\Omega)}$. Then $\hat{\boldsymbol{e}}$ is a unit eigenfunction of $T$ corresponding to the eigenvalue (cf. (5.2))

$$
\mu=(1+\lambda)^{-1}
$$


and we have

$$
\left|\mu-\mu_{h, \ell}\right|=\left|(T \hat{\boldsymbol{e}}, \hat{\boldsymbol{e}})-\left(T_{h} \boldsymbol{x}, \boldsymbol{x}\right)\right| \leq\left|\left(\left(T-T_{h}\right) \boldsymbol{x}, \boldsymbol{x}\right)\right|+|(T \hat{\boldsymbol{e}}, \hat{\boldsymbol{e}})-(T \boldsymbol{x}, \boldsymbol{x})| .
$$

From (5.6) we have

$$
\left|\left(\left(T-T_{h}\right) \boldsymbol{x}, \boldsymbol{x}\right)\right| \leq C_{\epsilon} h^{2-\epsilon}
$$

and it follows from Pythagoras' theorem with respect to the inner product $(T \cdot, \cdot)$ that

$$
\begin{aligned}
|(T \hat{\boldsymbol{e}}, \hat{\boldsymbol{e}})-(T \boldsymbol{x}, \boldsymbol{x})| & =|(T \hat{\boldsymbol{e}}, \hat{\boldsymbol{e}})-(T \hat{\boldsymbol{x}}, \hat{\boldsymbol{x}})-(T \hat{\boldsymbol{y}}, \hat{\boldsymbol{y}})| \\
& \leq\left(1-\|\hat{\boldsymbol{x}}\|_{L_{2}(\Omega)}^{2}\right)(T \hat{\boldsymbol{e}}, \hat{\boldsymbol{e}})+(T \hat{\boldsymbol{y}}, \hat{\boldsymbol{y}}) .
\end{aligned}
$$

Combining (5.13), (5.14) and (5.18), we find

$$
|(T \hat{\boldsymbol{e}}, \hat{\boldsymbol{e}})-(T \boldsymbol{x}, \boldsymbol{x})| \leq C_{\epsilon} h^{4-\epsilon},
$$

which together with (5.16)-(5.17) implies

$$
\left|\mu-\mu_{h, \ell}\right| \leq C_{\epsilon} h^{2-\epsilon} .
$$

The estimate (5.8) follows from (5.12), (5.15) and (5.19).

Recall that the gap $\hat{\delta}(M, N)$ between two subspaces $M$ and $N$ of a normed linear space $\left(X,\|\cdot\|_{X}\right)$ is defined by (cf. [39])

$$
\hat{\delta}(M, N)=\max (\delta(M, N), \delta(N, M)),
$$

where

$$
\delta(M, N)=\sup _{\substack{x \in M \\\|x\|_{X}=1}} \inf _{y \in N}\|x-y\|_{X}
$$

Therefore the statements about the gap between $V_{\lambda}$ and $V_{h, \lambda}$ follow immediately from (5.9) and (5.10).

Remark 5.2. The compactness of the solution operator $T$ and the existence of the uniform estimates (5.6) and (5.7) greatly simplify the analysis of the Maxwell eigenproblem. These ingredients are absent from Maxwell spectral approximations based on the full curl-curl problem and hence their justifications are much more involved $[10,11,8,20,21,9,19,12]$.

6. Numerical Results. In this section, we report a series of numerical examples that corroborate our theoretical results. Besides the $L_{2}$ error $\left\|\boldsymbol{u}-\stackrel{\bullet}{u}_{h}\right\|_{L_{2}(\Omega)}$ and the energy error $\left\|\stackrel{\circ}{\boldsymbol{u}}-\stackrel{\circ}{\boldsymbol{u}}_{h}\right\|_{h}$, we also report the errors measured in the semi-norm $|\cdot|_{\text {curl }}$ defined by

$$
|\boldsymbol{v}|_{\text {curl }}=\left\|\nabla_{h} \times \boldsymbol{v}\right\|_{L_{2}(\Omega)}
$$

6.1. Source Problems. We first demonstrate the performance of our scheme for the source problems where we take $\alpha$ to be $-k^{2}$ for all the computations in this subsection.

In the first experiment, we check the convergence behavior of our numerical scheme $(3.1)$ on the square $(0,0.5)^{2}$ with uniform meshes, where the exact solution is

$$
\stackrel{\circ}{\boldsymbol{u}}=[y(y-0.5) \sin (k y), x(x-0.5) \cos (k x)]
$$


Convergence of the scheme (3.1) on the square $(0,0.5)^{2}$ with uniform meshes and exact solution $\stackrel{i}{\text { given by }(6.1)}$

\begin{tabular}{|c|c|c|c|c|c|c|}
\hline \multirow[t]{2}{*}{$h$} & $\left\|\stackrel{\circ}{\boldsymbol{u}}-\stackrel{\circ}{\boldsymbol{u}}_{h}\right\|_{L_{2}(\Omega)}$ & \multirow{2}{*}{ order } & \multirow{2}{*}{ 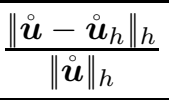 } & \multirow{2}{*}{ order } & \multirow{2}{*}{ 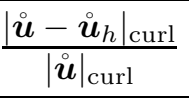 } & \multirow{2}{*}{ order } \\
\hline & $\|\stackrel{\sim}{\boldsymbol{u}}\|_{L_{2}(\Omega)}$ & & & & & \\
\hline \multicolumn{7}{|c|}{$k=0$} \\
\hline $1 / 10$ & $8.11 \mathrm{E}-02$ & - & $3.61 \mathrm{E}-01$ & - & $1.70 \mathrm{E}-01$ & - \\
\hline $1 / 20$ & $1.85 \mathrm{E}-02$ & 2.13 & $1.76 \mathrm{E}-01$ & 1.04 & $8.52 \mathrm{E}-02$ & 0.99 \\
\hline $1 / 40$ & $4.43 \mathrm{E}-03$ & 2.07 & $8.69 \mathrm{E}-02$ & 1.02 & $4.25 \mathrm{E}-02$ & 1.00 \\
\hline $1 / 80$ & $1.08 \mathrm{E}-03$ & 2.03 & $4.30 \mathrm{E}-02$ & 1.01 & $2.12 \mathrm{E}-02$ & 1.00 \\
\hline $1 / 160$ & $2.68 \mathrm{E}-04$ & 2.02 & $2.15 \mathrm{E}-02$ & 1.00 & $1.06 \mathrm{E}-02$ & 1.00 \\
\hline \multicolumn{7}{|c|}{$k=1$} \\
\hline $1 / 10$ & $7.19 \mathrm{E}-02$ & - & $3.45 \mathrm{E}-01$ & - & $1.64 \mathrm{E}-01$ & - \\
\hline $1 / 20$ & $1.65 \mathrm{E}-02$ & 2.12 & $1.68 \mathrm{E}-01$ & 1.04 & $8.14 \mathrm{E}-02$ & 1.01 \\
\hline $1 / 40$ & $3.93 \mathrm{E}-03$ & 2.07 & $8.32 \mathrm{E}-02$ & 1.02 & $4.09 \mathrm{E}-02$ & 1.00 \\
\hline $1 / 80$ & $9.60 \mathrm{E}-04$ & 2.04 & $4.13 \mathrm{E}-02$ & 1.01 & $2.05 \mathrm{E}-02$ & 1.00 \\
\hline $1 / 160$ & $2.37 \mathrm{E}-04$ & 2.01 & $2.06 \mathrm{E}-02$ & 1.01 & $1.02 \mathrm{E}-02$ & 1.00 \\
\hline \multicolumn{7}{|c|}{$k=10$} \\
\hline $1 / 10$ & $4.82 \mathrm{E}-01$ & - & $9.11 \mathrm{E}-01$ & - & $4.33 \mathrm{E}-01$ & - \\
\hline $1 / 20$ & $7.62 \mathrm{E}-02$ & 2.66 & $3.65 \mathrm{E}-01$ & 1.32 & $2.00 \mathrm{E}-01$ & 1.12 \\
\hline $1 / 40$ & $1.70 \mathrm{E}-02$ & 2.17 & $1.75 \mathrm{E}-01$ & 1.06 & $9.89 \mathrm{E}-02$ & 1.02 \\
\hline $1 / 80$ & $4.07 \mathrm{E}-03$ & 2.06 & $8.63 \mathrm{E}-02$ & 1.02 & $4.93 \mathrm{E}-02$ & 1.01 \\
\hline $1 / 160$ & $9.99 \mathrm{E}-04$ & 2.03 & $4.32 \mathrm{E}-02$ & 1.00 & $2.47 \mathrm{E}-02$ & 1.00 \\
\hline
\end{tabular}

for $k=0,1$ and 10. The results tabulated in Table 6.1 confirm the estimates (4.24) and (4.25). Observe also that, as expected, finer meshes are needed for computing satisfactory approximate solutions when the wave numbers become larger.

Since our numerical scheme is designed for solving the reduced curl-curl problem (1.5), its performance should not be affected by the addition to the right-hand side of a gradient term $\nabla G$ where $G \in H_{0}^{1}(\Omega)$. This is demonstrated by our second experiment. Here we add $\nabla G$ with

$$
G(x, y)=x y(x-0.5)(y-0.5) \sin (x+y)
$$

to the right-hand side

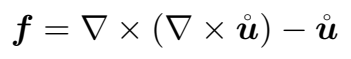

of the problem in the first experiment (with $k=1$ ) and compare their performance. The results are reported in Table 6.2.

Note that the scheme (3.1) differs from the one proposed in [15] because of the last two stabilizing terms. In the third experiment, we show that these two terms are necessary for the convergence of our method due to the lack of the continuity of the approximating functions across the element interfaces. See Table 6.3 for the numerical evidence.

The last example in this subsection demonstrates the convergence behavior of our scheme on the $L$-shaped domain $(-0.5,0.5)^{2} \backslash[0,0.5]^{2}$ with corners $(0.5,0),(0,0)$, $(0,0.5),(-0.5,0.5),(-0.5,-0.5)$ and $(0.5,-0.5)$. We take $k=1$ and the exact solution 
TABLE 6.2

Comparison of the performance of the scheme $(3.1)$ on $(0,0.5)^{2}$ with $k=1$ for the right-hand sides $\boldsymbol{f}$ and $\boldsymbol{f}+\nabla G$, where $\boldsymbol{f}$ is given by (6.3) and $G$ is given by (6.2)

\begin{tabular}{|c|c|c|}
\hline$h$ & $\mathrm{rhs}=f$ & rhs $=\boldsymbol{f}+\nabla G$ \\
\hline \multicolumn{3}{|c|}{$\left\|\boldsymbol{i}-\stackrel{\circ}{\boldsymbol{u}}_{h}\right\|_{L_{2}(\Omega)}$} \\
\hline $1 / 10$ & $\mathbf{0 . 0 0 1 6 4 3 0 9 2 6 6 1}$ & $\mathbf{0 . 0 0 1 6 4 3 0 2 0 3 6 3}$ \\
\hline $1 / 20$ & $\mathbf{0 . 0 0 0 3 7 6 0 0 1 6 0 7}$ & $\mathbf{0 . 0 0 0 3 7 5 9 9 2 4 0 6}$ \\
\hline $1 / 40$ & 0.000089765112 & 0.000089764564 \\
\hline $1 / 80$ & 0.000021922450 & 0.000021922530 \\
\hline $1 / 160$ & 0.000005415103 & 0.000005417204 \\
\hline \multicolumn{3}{|c|}{$\left\|\stackrel{\circ}{\boldsymbol{u}}-\stackrel{\circ}{\boldsymbol{u}}_{h}\right\|_{h}$} \\
\hline $1 / 10$ & $\mathbf{0 . 0 5 1 1 0 9} 229470$ & $\mathbf{0 . 0 5 1 1 0 9 7 9 2 1 6 9}$ \\
\hline $1 / 20$ & $\mathbf{0 . 0 2 4 9 1 9 7 6 0 8 3 2}$ & $\mathbf{0 . 0 2 4 9 1 9 9 1 1 6 6 4}$ \\
\hline $1 / 40$ & $\mathbf{0 . 0 1 2 2 9 6 8 8 0 7 6 5}$ & $\mathbf{0 . 0 1 2 2 9 6 9 5 2 9 8 6}$ \\
\hline $1 / 80$ & $\mathbf{0 . 0 0 6 1 0 7 3 2 3 5 6 6}$ & $\mathbf{0 . 0 0 6 1 0 7 3 6 2 3 4 3}$ \\
\hline $1 / 160$ & $\mathbf{0 . 0 0 3 0 4 3 4 0 0 5 3 0}$ & $\mathbf{0 . 0 0 3 0 4 3 4 2 4 4 6 4}$ \\
\hline
\end{tabular}

TABLE 6.3

Loss of convergence without the last two stabilizing terms in the scheme (3.1). The computation is on the square $(0,0.5)^{2}$ with uniform meshes and the exact solution $\boldsymbol{u}$ is given by (6.1) with $k=1$

\begin{tabular}{|c|c|c|c|c|}
\hline \multirow{2}{*}{$h$} & $\left\|\stackrel{\circ}{u}-\stackrel{\circ}{u}_{h}\right\|_{L_{2}(\Omega)}$ & \multirow{2}{*}{ order } & $\left|\stackrel{\boldsymbol{u}}{ }-\dot{\boldsymbol{u}}_{h}\right|_{\text {curl }}$ & \multirow{2}{*}{ order } \\
\hline & 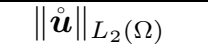 & & $|\stackrel{\circ}{u}|_{\text {curl }}$ & \\
\hline $1 / 10$ & $5.17 \mathrm{E}-00$ & - & $2.61 \mathrm{E}-01$ & - \\
\hline $1 / 20$ & $4.91 \mathrm{E}-00$ & 0.22 & $1.71 \mathrm{E}-01$ & 0.61 \\
\hline $1 / 40$ & $4.78 \mathrm{E}-00$ & 0.04 & $1.33 \mathrm{E}-01$ & 0.37 \\
\hline $1 / 80$ & $4.69 \mathrm{E}-00$ & 0.03 & $1.20 \mathrm{E}-01$ & 0.15 \\
\hline
\end{tabular}

is chosen to be

$$
\stackrel{\imath}{\boldsymbol{u}}=\nabla \times\left(r^{2 / 3} \cos \left(\frac{2}{3} \theta-\frac{\pi}{3}\right) \phi(r / 0.5)\right),
$$

where $(r, \theta)$ are the polar coordinates at the origin and the cut-off function is given by

$$
\phi(r)= \begin{cases}1 & r \leq 0.25 \\ -16(r-0.75)^{3} & \\ \times\left[5+15(r-0.75)+12(r-0.75)^{2}\right] & 0.25 \leq r \leq 0.75 \\ 0 & r \geq 0.75\end{cases}
$$

The meshes are graded around the re-entrant corner with the grading parameter equal to $1 / 3$. The results are tabulated in Table 6.4 and they agree with the estimates (4.24) and (4.25).

6.2. Maxwell Eigenproblem. In this subsection, we report the numerical results for the Maxwell eigenproblem.

In the first experiment, the computation is carried out on the square domain $(0, \pi)^{2}$ with uniform meshes. In this case, the exact eigenvalues are $r^{2}+s^{2}, r, s=$ $0,1,2,3,4, \cdots$ with $r^{2}+s^{2} \neq 0$. For instance, the first ten eigenvalues are $1,1,2,4,4$, 
TABLE 6.4

Convergence of the scheme (3.1) with graded meshes on the L-shaped domain $(-0.5,0.5)^{2} \backslash$ $[0,0.5]^{2}$ with $k=1$ and exact solution $\mathfrak{u}$ given by (6.4)

\begin{tabular}{|c|c|c|c|c|c|c|}
\hline$h$ & $\frac{\left\|\stackrel{\circ}{\boldsymbol{u}}-\stackrel{\circ}{\boldsymbol{u}}_{h}\right\|_{L_{2}(\Omega)}}{\|\stackrel{\circ}{\boldsymbol{u}}\|_{L_{2}(\Omega)}}$ & order & 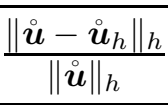 & order & 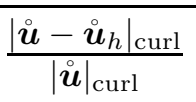 & order \\
\hline $1 / 4$ & $1.77 \mathrm{E}+02$ & - & $2.20 \mathrm{E}+01$ & - & $9.13 \mathrm{E}-00$ & - \\
\hline $1 / 8$ & $4.22 \mathrm{E}+01$ & 2.07 & $7.76 \mathrm{E}-00$ & 1.50 & $4.52 \mathrm{E}-00$ & 1.02 \\
\hline $1 / 16$ & $3.42 \mathrm{E}-00$ & 3.63 & $2.24 \mathrm{E}-00$ & 1.79 & $7.86 \mathrm{E}-01$ & 2.52 \\
\hline $1 / 32$ & $7.10 \mathrm{E}-01$ & 2.27 & $1.09 \mathrm{E}-00$ & 1.04 & $4.27 \mathrm{E}-01$ & 0.88 \\
\hline $1 / 64$ & $1.77 \mathrm{E}-01$ & 2.01 & $5.53 \mathrm{E}-01$ & 0.98 & $2.34 \mathrm{E}-01$ & 0.86 \\
\hline
\end{tabular}

5, 5, 8, 9, 9. In Figure 6.1, we plot the first twenty numerical eigenvalues versus the parameter $n=\pi / h$. The symbol "o" on the right side denotes the exact eigenvalue, and "(2)" indicates that the multiplicity of the eigenvalue is 2. All the numerical approximations converge at a second order rate. To save space, only the first five numerical eigenvalues are included in Table 6.5. Furthermore, there is no spurious eigenvalue in our results.

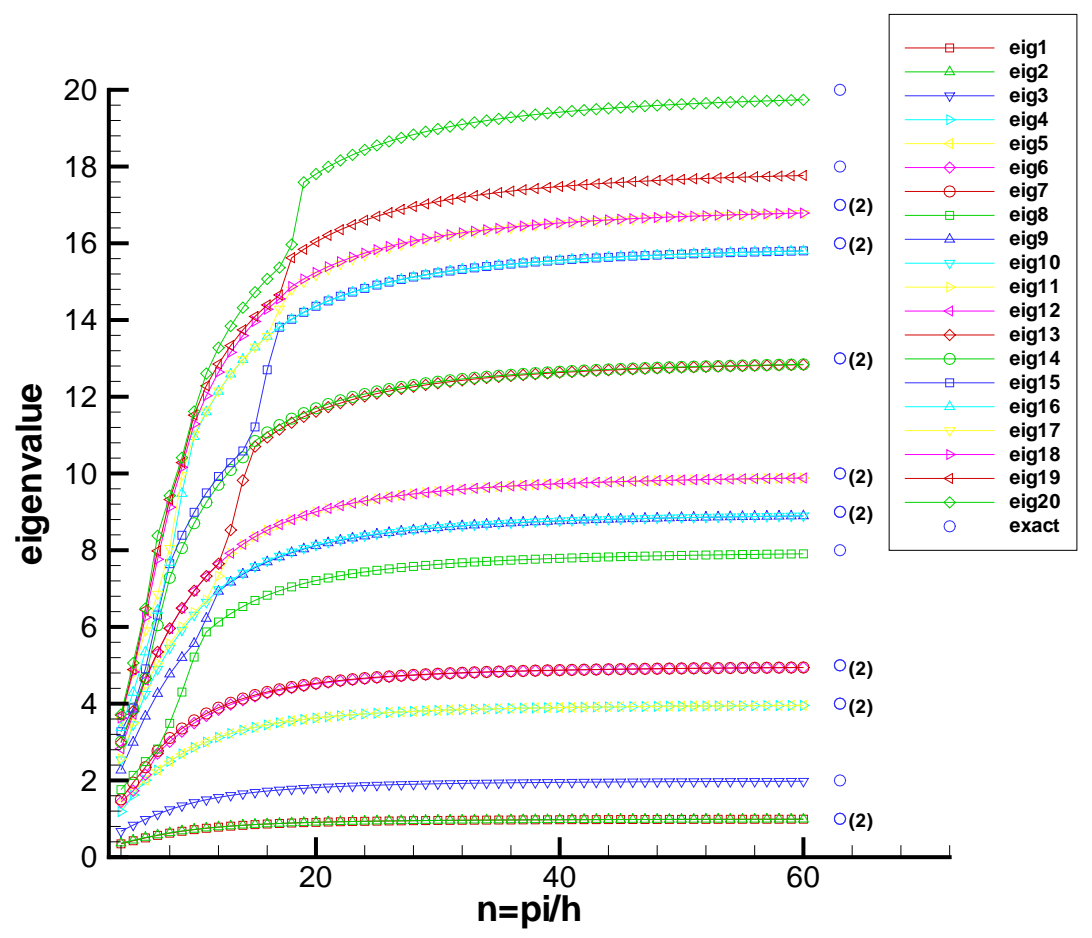

FIG. 6.1. First twenty numerical eigenvalues versus $n=\pi / h$ for Maxwell operator on $(0, \pi)^{2}$

In the second experiment, we compute the eigenvalues for the $L$-shaped domain $(-0.5,0.5)^{2} \backslash[0,0.5]^{2}$ and the meshes are graded around the re-entrant corner with the grading parameter equal to $1 / 3$. Table 6.6 contains the first five numerical eigenvalues, 
TABLE 6.5

Convergence of the first five numerical eigenvalues on $(0, \pi)^{2}$, with $\gamma=$ order of convergence and $\lambda=$ exact eigenvalue

\begin{tabular}{|c|c|c|c|c|c|c|c|c|c|c|}
\hline$h$ & $1^{\text {st }}$ & $\gamma$ & $2^{\text {nd }}$ & $\gamma$ & $3^{\text {rd }}$ & $\gamma$ & $4^{\text {th }}$ & $\gamma$ & $5^{\text {th }}$ & $\gamma$ \\
\hline$\pi / 4$ & 0.347 & - & 0.367 & - & 0.673 & - & 1.188 & - & 1.409 & - \\
$\pi / 8$ & 0.626 & 0.80 & 0.634 & 0.79 & 1.239 & 0.80 & 2.495 & 0.90 & 2.496 & 0.78 \\
$\pi / 16$ & 0.863 & 1.45 & 0.866 & 1.45 & 1.723 & 1.46 & 3.446 & 1.44 & 3.446 & 1.44 \\
$\pi / 32$ & 0.962 & 1.84 & 0.963 & 1.84 & 1.923 & 1.84 & 3.845 & 1.83 & 3.845 & 1.83 \\
$\pi / 64$ & 0.990 & 1.97 & 0.990 & 1.97 & 1.980 & 1.97 & 3.960 & 1.96 & 3.960 & 1.96 \\
$\pi / 128$ & 0.998 & 1.97 & 0.998 & 2.00 & 1.995 & 2.01 & 3.990 & 1.99 & 3.990 & 1.99 \\
\hline \hline$\lambda$ & 1 & & 1 & & 2 & & 4 & & 4 & \\
\hline
\end{tabular}

which show second order convergence of our method. This agrees with our analysis. In this case the "exact eigenvalues" are derived from numerical results of Dauge, which can be found at http://perso.univ-rennes1.fr/monique.dauge/core/index.html. In Figure 6.2, we plot the first ten numerical eigenvalues versus the parameter $n=$ $1 / 2 h$. Again, there is no spurious eigenvalue.

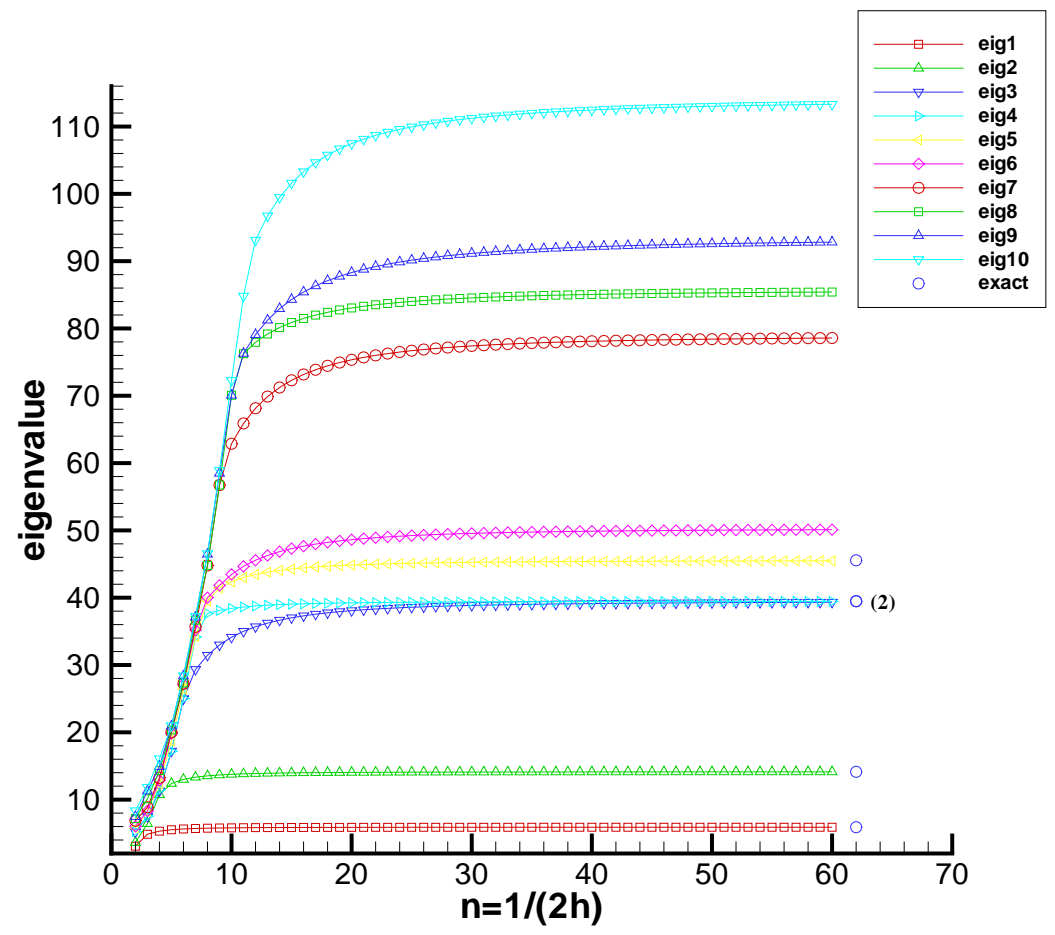

Fig. 6.2. First ten numerical eigenvalues versus $n=1 / 2 h$ for the Maxwell operator on $(-0.5,0.5)^{2} \backslash[0,0.5]^{2}$.

7. Concluding Remarks. We have analyzed an interior penalty method with weak over-penalization for the two-dimensional reduced curl-curl problem and the 
TABLE 6.6

Convergence of the first five numerical eigenvalues on the L-shaped domain $(-0.5,0.5)^{2} \backslash[0,0.5]^{2}$ with graded meshes, $\mu=1 / 3$ at the re-entrant corner, $\gamma=$ order of convergence and $\lambda=$ exact eigenvalue

\begin{tabular}{|c|c|c|c|c|c|c|c|c|c|c|}
\hline$h$ & $1^{\text {st }}$ & $\gamma$ & $2^{\text {nd }}$ & $\gamma$ & $3^{\text {rd }}$ & $\gamma$ & $4^{\text {th }}$ & $\gamma$ & $5^{\text {th }}$ & $\gamma$ \\
\hline $1 / 4$ & 2.626 & - & 3.442 & - & 5.087 & - & 5.174 & - & 5.656 & - \\
$1 / 8$ & 4.747 & 1.50 & 9.963 & 1.36 & 11.233 & 0.28 & 11.316 & 0.28 & 12.226 & 0.26 \\
$1 / 16$ & 5.574 & 1.81 & 13.102 & 2.01 & 30.631 & 1.67 & 36.653 & 3.32 & 38.548 & 2.25 \\
$1 / 32$ & 5.819 & 1.98 & 13.875 & 1.98 & 37.008 & 1.84 & 38.822 & 2.11 & 44.091 & 2.26 \\
$1 / 64$ & 5.882 & 2.02 & 14.071 & 2.00 & 38.847 & 1.97 & 39.319 & 2.04 & 45.204 & 2.05 \\
\hline \hline$\lambda$ & 5.902 & & 14.136 & & 39.478 & & 39.478 & & 45.558 & \\
\hline
\end{tabular}

Maxwell eigenproblem. This method is inconsistent but stable without the need of tuning any penalty parameter. We expect other discontinuous Galerkin schemes will be discovered along this line. An advantage of this new approach is that fast solvers for the resulting schemes can be constructed using techniques developed for elliptic problems.

The scheme developed in this paper can be extended to three dimensional domains. In the case of uniform meshes, our two dimensional analysis can be easily generalized to establish convergence at a sub-optimal rate. It is likely that quasioptimal convergence rates can be recovered using graded meshes, which is of course much more complicated in three dimensions $[2,3]$. This will be one of the topics of our ongoing research.

Acknowledgement. The first author would like to thank the Alexander von Humboldt Foundation for support through her Humboldt Research Award. Part of the research in this paper was carried out while the first and third authors were visiting the Humboldt Universität zu Berlin and they would like to thank the members of the Institut für Mathematik for their hospitality.

\section{REFERENCES}

[1] Th. Apel. Anisotropic Finite Elements. Teubner, Stuttgart, 1999.

[2] Th. Apel and S. Nicaise. The finite element method with anisotropic mesh grading for elliptic problems in domains with corners and edges. Math. Methods Appl. Sci., 21:519-549, 1998.

[3] Th. Apel, S. Nicaise, and J. Schöberl. Crouzeix-Raviart type finite elements on anisotropic meshes. Numer. Math., 89:193-223, 2001.

[4] Th. Apel, A.-M. Sändig, and J.R. Whiteman. Graded mesh refinement and error estimates for finite element solutions of elliptic boundary value problems in non-smooth domains. Math. Methods Appl. Sci., 19:63-85, 1996.

[5] F. Assous, P. Ciarlet, Jr., S. Labrunie, and S. Lohrengel. The singular complement method. In N. Debit, M. Garbey, R. Hoppe, D. Keyes, Y. Kuznetsov, and J. Périaux, editors, Domain Decomposition Methods in Science and Engineering, pages 161-189. CIMNE, Barcelona, 2002.

[6] F. Assous, P. Ciarlet, Jr., and E. Sonnendrücker. Resolution of the Maxwell equation in a domain with reentrant corners. M2AN Math. Model. Numer. Anal., 32:359-389, 1998.

[7] I. Babuška and J. Osborn. Eigenvalue Problems. In P.G. Ciarlet and J.L. Lions, editors, Handbook of Numerical Analysis II, pages 641-787. North-Holland, Amsterdam, 1991.

[8] D. Boffi. Fortin operators and discrete compactness for edge elements. Numer. Math., 87:229246, 2000.

[9] D. Boffi. Approximation of eigenvalues in mixed form, discrete compactness property, and application to $h p$ mixed finite elements. preprint, 2005. 
[10] D. Boffi, F. Brezzi, and L. Gastaldi. On the convergence of eigenvalues for mixed formulations. Ann. Scuola Norm. Sup. Pisa Cl. Sci. (4), 25:131-154, 1997.

[11] D. Boffi, P. Fernandes, L. Gastaldi, and I. Perugia. Computational models of electromagnetic resonators: Analysis of edge element approximation. SIAM J. Numer. Anal., 36:1264-1290, 1999.

[12] D. Boffi, F. Kikuchi, and J. Schöberl. Edge element computation of Maxwell's eigenvalues on general quadrilateral meshes. Math. Models Methods Appl. Sci., 16:265-273, 2006.

[13] A. Bossavit. Computational Electromagnetism. Academic Press, San Diego, 1998.

[14] S.C. Brenner and C. Carstensen. Finite Element Methods. In E. Stein, R. de Borst, and T.J.R. Hughes, editors, Encyclopedia of Computational Mechanics, pages 73-118. Wiley, Weinheim, 2004.

[15] S.C. Brenner, F. Li, and L.-Y. Sung. A locally divergence-free nonconforming finite element method for the time-harmonic Maxwell equations. Math. Comp., 76:573-595, 2007.

[16] S.C. Brenner and L. Owens. A weakly over-penalized nonsymmetric interior penalty method. 2006 (to appear in Journal of Numerical Analysis, Industrial and Applied Mathematics).

[17] S.C. Brenner, L. Owens, and L.-Y. Sung. A weakly over-penalized symmetric interior penalty method (in preparation). 2007.

[18] S.C. Brenner and L.R. Scott. The Mathematical Theory of Finite Element Methods (Second Edition). Springer-Verlag, New York-Berlin-Heidelberg, 2002.

[19] A. Buffa and I. Perugia. Discontinuous Galerkin approximation of the Maxwell eigenproblem. SIAM J. Numer. Anal., 44:2198-2226, 2006.

[20] S. Caorsi, P. Fernandes, and M. Raffetto. On the convergence of Galerkin finite element approximations of electromagnetic eigenproblems. SIAM J. Numer. Anal., 38:580-607, 2000.

[21] S. Caorsi, P. Fernandes, and M. Raffetto. Spurious-free approximations of electromagnetic eigenproblems by means of Nédélec-type elements. M2AN, 35:331-358, 2001.

[22] P.G. Ciarlet. The Finite Element Method for Elliptic Problems. North-Holland, Amsterdam, 1978.

[23] M. Costabel. A remark on the regularity of solutions of Maxwell's equations on Lipschitz domains. Math. Methods Appl. Sci., 12:36-368, 1990.

[24] M. Costabel. A coercive bilinear form for Maxwell's equations. J. Math. Anal. Appl., 157:527$541,1991$.

[25] M. Costabel and M. Dauge. Maxwell and Lamé eigenvalues on polyhedra. Math. Methods Appl. Sci., 22:243-258, 1999.

[26] M. Costabel and M. Dauge. Singularities of electromagnetic fields in polyhedral domains. Arch. Ration. Mech. Anal., 151:221-276, 2000.

[27] M. Costabel and M. Dauge. Weighted regularization of Maxwell equations in polyhedral domains. Numer. Math., 93:239-277, 2002.

[28] M. Costabel, M. Dauge, and S. Nicaise. Singularities of Maxwell interface problems. $M^{2} A N$, 33:627-649, 1999.

[29] M. Costabel, M. Dauge, and C. Schwab. Exponential convergence of hp-FEM for Maxwell equations with weighted regularization in polygonal domains. Math. Models Methods Appl. Sci., 15:575-622, 2005.

[30] M. Crouzeix and P.-A. Raviart. Conforming and nonconforming finite element methods for solving the stationary Stokes equations I. RAIRO Anal. Numér., 7:33-75, 1973.

[31] P. Grisvard. Elliptic Problems in Non Smooth Domains. Pitman, Boston, 1985.

[32] C. Hazard and S. Lohrengel. A singular field method for Maxwell's equations: Numerical aspects for 2D magnetostatics. SIAM J. Numer. Anal., 40:1021-1040, 2002.

[33] R. Hiptmair. Finite elements in computational electromagnetism. Acta Numer., 11:237-339, 2002.

[34] P. Houston, I. Perugia, A. Schneebeli, and D. Schötzau. Interior penalty method for the indefinite time-harmonic Maxwell equation. Numer. Math., 100:485-518, 2005.

[35] P. Houston, I. Perugia, A. Schneebeli, and D. Schötzau. Mixed discontinuous Galerkin approximation of of the Maxwell operator: the indefinite case. M2AN, 39:727-753, 2005.

[36] P. Houston, I. Perugia, and D. Schötzau. Mixed discontinuous Galerkin approximation of of the Maxwell opertor. SIAM J. Numer. Anal., 42:434-459, 2004.

[37] P. Houston, I. Perugia, and D. Schötzau. Mixed discontinuous Galerkin approximation of of the Maxwell operator: non-stabilized formulation. J. Sci. Comput., 22/23:315-346, 2005.

[38] J.-M. Jin. The Finite Element Method in Electromagnetics (Second Edition). John Wiley \& Sons, Inc., New York, 2002.

[39] T. Kato. Perturbation Theory of Linear Operators. Springer-Verlag, Berlin, 1966.

[40] P. Monk. Finite Element Methods for Maxwell's Equations. Oxford University Press, New 
York, 2003.

[41] J.-C. Nédélec. Mixed finite elements in $\mathbf{R}^{3}$. Numer. Math., 35:315-341, 1980.

[42] J.-C. Nédélec. A new family of mixed finite elements in $\mathbf{R}^{3}$. Numer. Math., 50:57-81, 1986.

[43] I. Perugia, D. Schötzau, and P. Monk. Stabilized interior penalty methods for the time-harmonic Maxwell equations. Comput. Methods. Appl. Mech. Engrg., 191:4675-4697, 2002.

[44] A. Schatz. An observation concerning Ritz-Galerkin methods with indefinite bilinear forms. Math. Comp., 28:959-962, 1974. 